\title{
Effect of Fungi on the Destruction of Historical Parchment and Paper Documents
}

\author{
Hanna Kwaśna ${ }^{1 *}$, Joanna Karbowska-Berent ${ }^{2}$, Jolanta Behnke-Borowczyk ${ }^{1}$ \\ ${ }^{1}$ Poznań University of Life Sciences, Department of Forest Pathology, Poznań, Poland \\ ${ }^{2}$ Nicolaus Copernicus University, Faculty of Fine Arts, Torun, Poland
}

Received: 9 May 2019

Accepted: 24 July 2019

\begin{abstract}
Fungal frequency and diversity were studied in historical, parchment and paper documents using Illumina sequencing. In total, 156 494, 52451 and 41615 sequences were obtained from three samples. Documents were colonized by 225 taxa. Glomeromycota, Zygomycota, Ascomycota and Basidiomycota were represented by 1, 8, 131 and 83 taxa, respectively. Fungal communities included plant pathogens, epiphytes or endophytes of a wide range of plants and possibly opportunistic plant pathogens, saprotrophs common in soil, on decaying leaves, needles, wood and on other plant material, human pathogens, animal and nematode pathogens, entomopathogenic taxa, mycoparasites, white and black yeast-like taxa, taxa with antagonistic and medicinal properties, lichenized fungi, food contaminants, common contaminants of indoor, built environments, taxa on herbivore dung, keratin-degrading taxa, xerophilic taxa, and an endangered fungi included in the Red List of Threatened Species. A non-destructive and non-invasive method for quantitative characterization of parchment deterioration, based on spectral measurements, was used for evaluating the scale of damage.The collagen-to-gelatin ratio, estimated from the synchronous fluorescence spectra of the studied samples and of pure collagen and gelatin, was suggested for characterizing parchment condition. Analysis of fluorescence peaks indicated the moderate stage of deterioration of the studied documents.
\end{abstract}

Keywords: diversity, fungi, ITS1/2 rDNA, historical documents, parchment

\section{Introduction}

Microorganisms (bacteria, archaea and fungi), together with lichens and insects, due to their biodeteriorative potential, damage items of cultural heritage. Fungi play an important role in archives and libraries where stored objects are made of paper or

*e-mail: hanna.kwasna@up.poznan.pl parchment. The main component of paper is cellulose, and of parchment it is inner collagen type I, with its typical fibre arrangement and external gelatin layers. Paper and parchment can be sources of energy, carbon and nitrogen for numerous microorganisms.

Bacteria are the main contaminants of parchment due to their preferences for higher $\mathrm{pH}$ [1-6]. Filamentous fungi also play an important role in biodeterioration due to their preferences for lower temperature and higher humidity, often present in archives. Typical fungal deterioration is caused by the slowgrowing, often mitosporic, cellulolitic or proteolytic 
and often xerophilic Ascomycetes from the genera Alternaria, Aspergillus, Cladosporium, Chaetomium, Chrysosporium, Paecilomyces, Penicillium, Phoma or Stachybotrys [5, 7-16]. On contaminated objects, fungi produce coloured stains (species of Chaetomium, Epicoccum, Monoascus), and emit strong odours (Trichoderma spp.) or toxic compounds (Stachybotrys spp.).

Fungal contamination usually arises from airborne inoculum. Colonization is determined by the nature and chemical composition of the material, climate, conditions of storage (i.e., poor ventilation and temperature changes, which cause water condensation), the mode and frequency of document usage and methods of cleaning.

Deterioration may cause distortion and deformation of documents. They become misshapen, the text fades, and contaminated areas may be covered with white films and multi-coloured spots and residues, called 'foxing' [17].

Biodeterioration and mechanical damage result from chemical degradation by fungal enzymes (cellulases, collagenases), chemical modification of inorganic components and production of pigments and organic acids penetrating and discoloring the document structure $[10,15,18,19]$.

The need for prevention and correct protection of historical objects is emphasized by conservators, archivists, and museologists [11, 20]. Implementation of appropriate remedial measures must be preceded by detailed research, including detection and recognition of the potential microbial hazard.

The aims of this study were: (i) to determine the abundance and diversity of fungi in historic parchment and paper documents in two archives in Poland, and (ii) to consider the destructive activity of the fungi detected in the prevailing environmental conditions.

Detection and identification of fungi was based on fungal genomic ITS rDNA studied with the Illumina technique. The ITS rDNA sequencing for studies of fungal contamination on historical documents was applied earlier [15, 21].

\section{Materials and Methods}

\section{Description of Documents}

Analyses of three samples from two documents were made in 2016.

Document 1 is a book by Ioannes de Pineda SI entitled "Ad suos in Salomonem commentarios ... libri octo", printed in 1613 in Mainz, Germany. Until 1881 the book was in a cloister in the Warmian-Masurian Voivodeship in northeastern Poland. In 1882 the book was moved to an attic of the parish building and stored in a wooden cabinet, with seasonal variations in temperature and humidity. Currently the book is stored in the Old Documents Archive in Torun Diocese. Its binding is made of cardboard covered with parchment. Its pages are made of handmade paper. The page edges are dyed with ultramarine. The book shows symptoms of deformation and fungal contamination (powdery or fluffy white, grey, reddish, brown or black deposits in numerous places on the binding and pages). The text is faded.

Document 2 is a torah originating from the $19^{\text {th }}$ century from Zamość County in eastern Poland. In 1940-1970, wrapped in paper, it was stored in a wooden cabinet with exposure to seasonal variations of temperature and humidity. Since 2014 it has been stored in acid-free paper in a wood and glass cabinet in the storeroom of the Foundation for the Preservation of Jewish Heritage in Poland, in Warsaw. Its scroll is made of calf parchment treated with chalk and polished. Two wooden shafts are made of maple wood. Four crowns attached to shafts are made of lime wood. The wooden elements are polychromed and dyed with orpiment, ultramarine, English red, red lead, white, green and black natural paints and calcium carbonate. The handwriting on the scroll is done with a metaltannic ink. Defects have been repaired with calfskin glued with gum Arabic or joined with natural or partly dyed linen threads. The torah parchment is creased and cracked vertically and horizontally. Its deterioration occurs mostly in gum Arabic-treated parts. There is no visible fungal contamination.

\section{Quantitative Characterization of Parchment Deterioration}

Both documents and the control currently made parchment sample were independently assessed by spectral measurements at $25^{\circ} \mathrm{C}$ [22]. The fluorescence was examined with a spectro-fluorimeter equipped with a surface-analysis accessory. Spectra were measured from both sides. The excitation wavelengths were $243 \mathrm{~nm}$ and $298 \mathrm{~nm}$. The excitation and emission bandwidths were $4 \mathrm{~nm}$. Fluorescence was correlated with deterioration of documents. Quantitative analysis was based on the comparison of spectra of collagen, gelatin and our samples. The collagen-to-gelatin ratio $(\mathrm{C} / \mathrm{G}$ ratio) and the percentage of collagen were estimated.

\section{Sampling for Mycological Analysis}

Samples 1 and 2 from Document 1 were respectively from white, fluffy deposits present on the parchment of the book binding and from greyish powdery deposits present on the book pages. Sample 3 from Document 2 was from cream-beige-brown spots covered with powdery deposits present on gum Arabictreated parts of the torah scroll. Samples were collected with a sterile scalpel from the surface of $1 \mathrm{~cm}^{2}$ areas with no text. 


\section{Molecular Identification \\ DNA Extraction, Amplification and Sequencing}

DNA was extracted with a Bead-Beat Micro AX Gravity Kit (A \& A Biotechnology). ITS 1/2 rDNA amplification was performed with fungus-specific primers gITS7 (5' GTG ART CAT CGA RTC TTT G 3) [23] and iITS4 (5`TCC TCC GCT TAT TGATAT GC 3). The PCR reaction mixture $(25 \mu \mathrm{l})$ consisted of $12.5 \mu \mathrm{l}$ of $2 \mathrm{x}$ MixPCR, $0.2 \mu \mathrm{M}$ of each primer, $1.5 \mu 1$ of purified and diluted DNA and $10.6 \mu \mathrm{l}$ of water. The PCR reaction was performed under the following conditions: denaturation at $94^{\circ} \mathrm{C}$ for $5 \mathrm{~min}$, followed by 35 cycles of denaturation at $94^{\circ} \mathrm{C}$ for $30 \mathrm{~s}$, annealing at $56^{\circ} \mathrm{C}$ for $30 \mathrm{~s}$ and elongation at $72^{\circ} \mathrm{C}$ for $30 \mathrm{~s}$, and a final elongation at $72^{\circ} \mathrm{C}$ for $7 \mathrm{~min}$. The amplicons were sequenced using the Illumina technique.

\section{Bioinformatics Analysis}

A table of operational taxonomic units (OTUs) was prepared by PIPITS, version 1.2.0 [24]. Read-pairs were joined with PEAR, version 0.9.6 [25], filtered with a quality threshold of $\mathrm{q}=30$ by FASTX-toolkit, version 0.0 .13 (http://hannonlab.cshl.edu/fastx toolkit/ index.html), converted to Fasta format and merged into a single file. Prepared sequences were dereplicated and subregions of ITS were selected with the use of ITSx, version 1.0.11 [26]. Unique sequences and those shorter than $100 \mathrm{bp}$ were removed. Remaining sequences were clustered with $97 \%$ sequence identity. The resulting representative sequences for each cluster were subjected to chimera detection and removal using the UNITE UCHIME reference dataset, version 6.0 (https://unite.ut.ee/index.php). The input sequences were then mapped onto the representative sequences and taxonomy assigned using an RDP Classifier, version 2.10.2 against UNITE fungal ITS reference database, version 11.2 [27]. Sequences were identified by comparison with reference sequences from the National Center for Biotechnology Information (NCBI) database. The abundance of fungi was defined as the number of OTUs in a sample. Frequency of an individual taxon was defined as the percentage (\%) of OTUs in the total number of OTUs. Diversity was defined as the number of species in a sample.

\section{Statistical Analysis}

Diversity in microbial communities was calculated for each community. Analyses were based on the abundance of fungi and taxonomic composition of communities [28]. Diversity is indicated by Margalef's diversity index (DMg) and Shannon's diversity index $\left(H^{\prime}\right)$. Evenness and dominance are indicated by Simpson's diversity index (D), Shannon's evenness index (E) and Berger-Parker's dominance index (d). The similarity between fungal communities is determined by Sorensen's qualitative similarity index (CN).
Similarity and relationships among fungal communities are shown by a heat map and Venn diagram (Fig. 1, Table 1).

\section{Results}

Compared to the control, aging and deterioration of documents caused a decrease in fluorescence intensity, the spectral shift of the main peak and an overall change in the fluorescence spectral features. For $298 \mathrm{~nm}$ excitation samples 1-3 overall fluorescence peaks were at $440-460 \mathrm{~nm}$, and control sample fluorescence peaked at $400 \mathrm{~nm}$. For $243 \mathrm{~nm}$ excitation the second broad weak bands of samples 1-3 emerged at 400-500 nm, and control sample fluorescence peak was at $300 \mathrm{~nm}$. The spectrum of collagen consisted of two well-defined peaks at 305 and $345 \mathrm{~nm}$ and an additional broad band with a maximum at $440 \mathrm{~nm}$. The spectrum of gelatin has a well-defined peak at $440 \mathrm{~nm}$ and additional broad bands at 305 and $345 \mathrm{~nm}$. Spectra of samples 1 and 3 shifted toward pure gelatin. The $\mathrm{C} / \mathrm{G}$ ratio in sample 1 was $0.8-8.2$ and in sample 3 was 1.2-8.0. The average percentage of collagen in samples 1 and 3 was 8.9 and 9.1.

Three samples yielded, respectively, 156 494, 52451 and 41615 raw sequences (Table 1). There were 156 $393=99.93 \%, 52355=99.81 \%$ and $40689=97.77 \%$ sequences of culturable fungi; $9=0.006 \%, 37=0.071 \%$ and $548=1.317 \%$ sequences of non-culturable fungi; and $37=0.024 \%, 19=0.036 \%$ and $375=0.901 \%$ sequences of organisms with no reference sequence in NCBI.

Documents were colonized by at least 225 fungal taxa (Table 1). Glomeromycota, Zygomycota, Ascomycota and Basidiomycota were represented

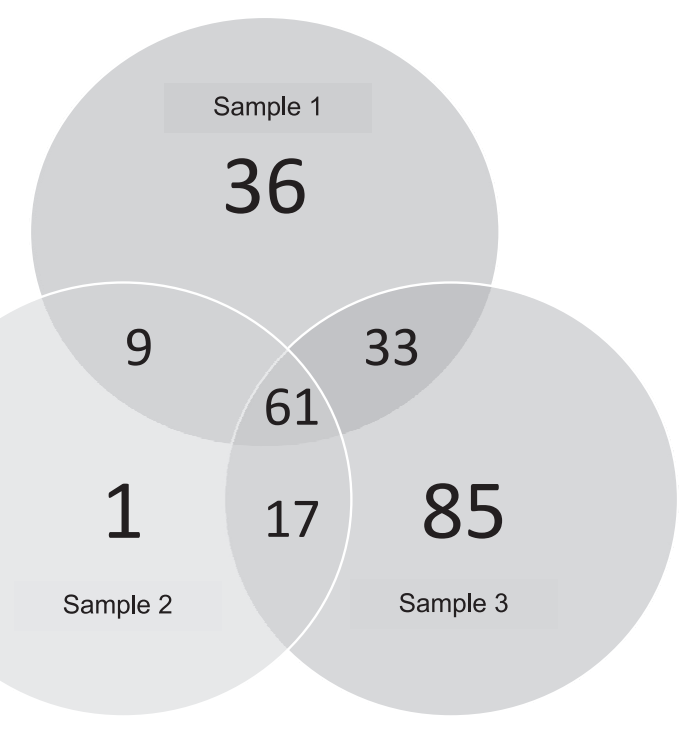

Fig. 1. Venn diagram: overlapping circles illustrate the similarities, differences, and relationships between fungal communities, with numbers of taxa shown. 


\begin{tabular}{|c|c|c|c|c|c|c|c|c|c|c|c|c|c|c|c|c|}
\hline \multirow{2}{*}{ 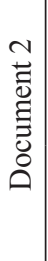 } & \multirow{2}{*}{ 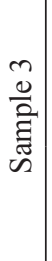 } & \multirow{2}{*}{ 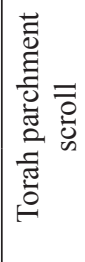 } & 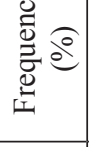 & 0 & 0 & 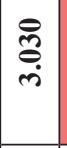 & $\tilde{c}$ & ठे & 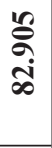 & $\stackrel{\infty}{0}$ & $\stackrel{\circlearrowright}{\Xi}$ & $\frac{\tilde{6}}{0}$ & $\tilde{\delta}$ & $\frac{7}{0}$ & $\exists$ & 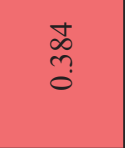 \\
\hline & & & $\dot{z}$ & 0 & 0 & $\mid \begin{array}{l}\vec{J} \\
\Xi\end{array}$ & $\underset{\infty}{\stackrel{f}{f}}$ & $\stackrel{\nabla}{\vec{\gamma}}$ & 姁 & 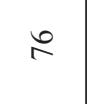 & ్్ㅇ & 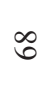 & $\stackrel{\infty}{\stackrel{\infty}{\sigma}}$ & in & $\stackrel{\text { }}{\stackrel{2}{\gamma}}$ & $\stackrel{8}{8}$ \\
\hline \multirow{4}{*}{$\theta$} & \multirow{2}{*}{ 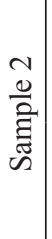 } & \multirow{2}{*}{ 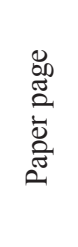 } & 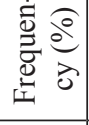 & 0 & 0 & $\mid$ & $\stackrel{\infty}{\stackrel{\infty}{+}}$ & $\stackrel{4}{\stackrel{0}{0}}$ & $\begin{array}{l}\stackrel{0}{\hat{N}} \\
\stackrel{2}{2}\end{array}$ & 홍 & 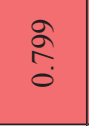 & 0 & $\stackrel{\infty}{=}$ & $\begin{array}{l}\infty \\
\stackrel{\infty}{\infty} \\
0\end{array}$ & $\underset{\infty}{\stackrel{y}{\infty}}$ & $\stackrel{0}{0}$ \\
\hline & & & $\dot{z}$ & 0 & 0 & 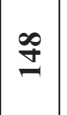 & 6 & $\bar{\infty}$ & 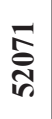 & m & $\vec{\gamma}$ & 0 & $\widetilde{\sigma}$ & fo & 竎 & $\nabla$ \\
\hline & \multirow{2}{*}{$\begin{array}{l}\overrightarrow{0} \\
\text { 离 } \\
\text { ch }\end{array}$} & \multirow{2}{*}{ 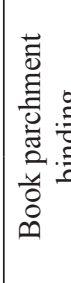 } & 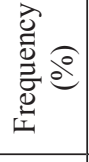 & 产 & $\overline{8}$ & $\mid$ & $\begin{array}{l}\stackrel{8}{0} \\
0\end{array}$ & $\begin{array}{l}\overrightarrow{8} \\
i\end{array}$ & 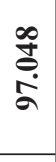 & $\stackrel{\vec{J}}{\stackrel{m}{=}}$ & $\stackrel{8}{+}$ & @े & 客 & ڤे & $\begin{array}{l}\dot{a} \\
\dot{\infty}_{0}\end{array}$ & \& \\
\hline & & & $\dot{z} \dot{D}_{0}^{n}$ & - & - & $\underset{\tilde{m}}{ \pm}$ & $a$ & तె & $\begin{array}{l}n \\
\infty \\
\frac{1}{n}\end{array}$ & 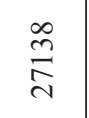 & $\underset{\mathbb{U}}{\infty}$ & $\Xi$ & $r$ & సิ & $\begin{array}{l}\hat{\widehat{0}} \\
\text { ปे }\end{array}$ & 6 \\
\hline \multicolumn{4}{|c|}{ 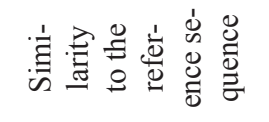 } & & ڤ̊ํㅁ & & مٌ & ลे & & ลें & ลें & ลें & छें & $\frac{\stackrel{\circ}{\circ}}{\stackrel{1}{a}}$ & ळ & छे \\
\hline \multicolumn{4}{|c|}{$\frac{\overrightarrow{0}}{\tilde{c}}$} & & 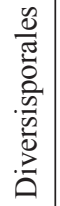 & & 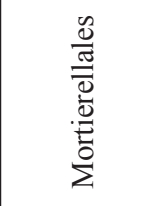 & 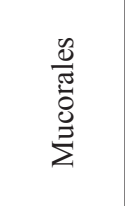 & & 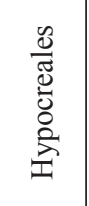 & $\begin{array}{l}\frac{0}{0} \\
\frac{0}{\pi} \\
\frac{0}{0} \\
\frac{0}{0} \\
\frac{0}{2} \\
\frac{0}{2}\end{array}$ & 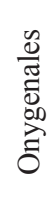 & 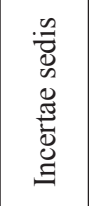 & & 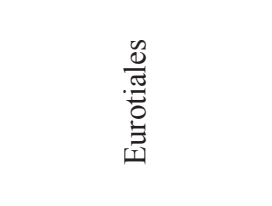 & $\begin{array}{l}\frac{0}{0} \\
\frac{0}{00} \\
\frac{0}{0} \\
\overline{0} \\
0\end{array}$ \\
\hline & & 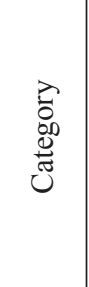 & & & & & 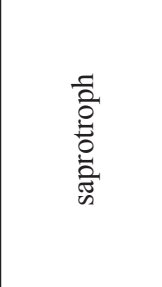 & 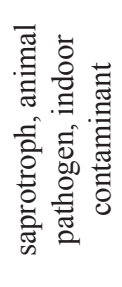 & & 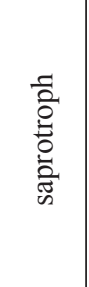 & 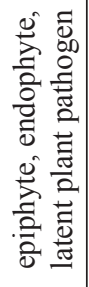 & 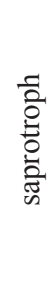 & 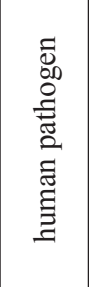 & & 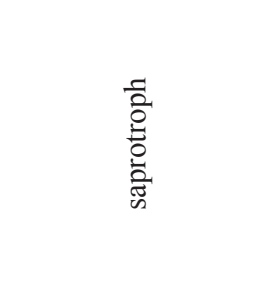 & 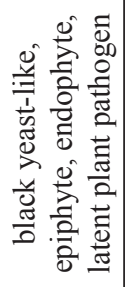 \\
\hline \multicolumn{4}{|c|}{ 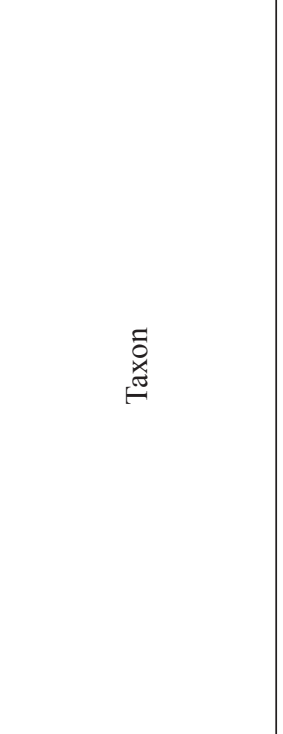 } & 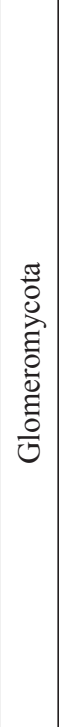 & 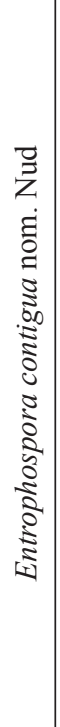 & 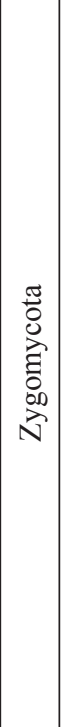 & 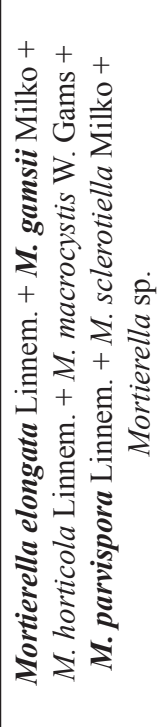 & 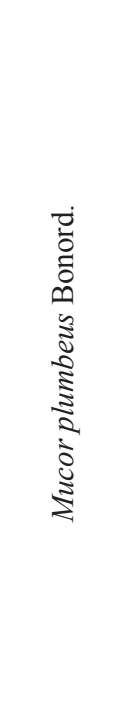 & 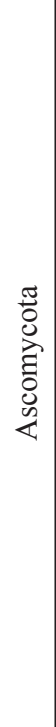 & 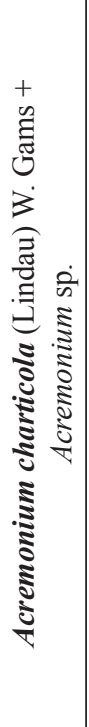 & 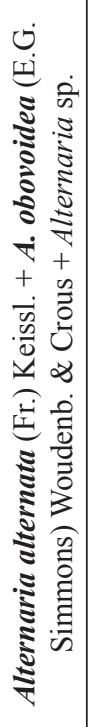 & 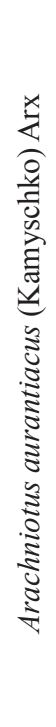 & 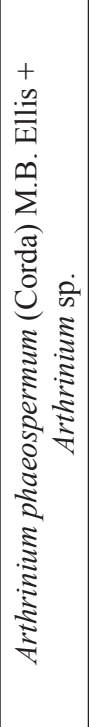 & 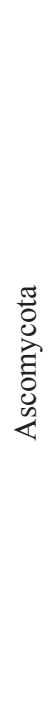 & 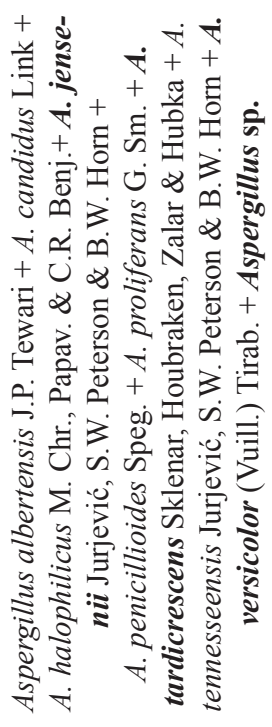 & 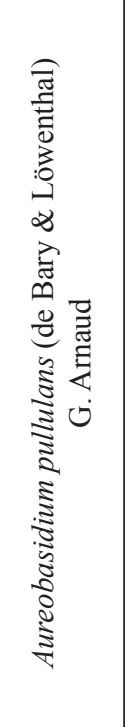 \\
\hline \multicolumn{4}{|c|}{$\dot{\dot{z}}$} & & $-\dot{ }$ & & $-i$ & $i$ & & $\dot{-}$ & $i$ & $\dot{m}$ & $\dot{+}$ & in & $0^{\circ}$ & $\therefore$ \\
\hline
\end{tabular}




\begin{tabular}{|c|c|c|c|c|c|c|c|c|c|c|c|c|c|c|c|c|c|c|c|}
\hline 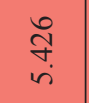 & $\frac{m}{i}$ & $\begin{array}{l}\vec{\exists} \\
\dot{\sigma}\end{array}$ & \begin{tabular}{l}
\multicolumn{2}{c}{} \\
$\stackrel{a}{a}$
\end{tabular} & $\begin{array}{l}0 \\
0 \\
0 \\
0\end{array}$ & $\begin{array}{l}\text { ஃे } \\
\stackrel{0}{0}\end{array}$ & 0 & $\begin{array}{l}\tilde{a} \\
\stackrel{0}{0}\end{array}$ & $\begin{array}{l}\stackrel{2}{ } \\
\infty \\
\dot{r}\end{array}$ & $\begin{array}{l}\overrightarrow{\tilde{F}} \\
\dot{\sim}\end{array}$ & $\begin{array}{l}\text { ¿े. } \\
\text { ¿े }\end{array}$ & 0 & ల్ల & ô. & $\frac{\tilde{\rho}}{0}$ & $\Xi$ & $\stackrel{n}{\stackrel{2}{0}}$ & $\vec{\nabla}$ & 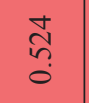 & 0 \\
\hline 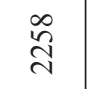 & $\overrightarrow{\vec{\nabla}}$ & $\Xi$ & $\stackrel{\mathbb{N}}{\tilde{6}}$ & 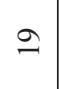 & 우 & 0 & $n$ & $\stackrel{0}{0}$ & $\underset{\infty}{+}$ & $\simeq$ & 0 & in & 兮 & $\stackrel{f}{f}$ & fo & $\cong$ & $=$ & $\stackrel{\infty}{\vec{v}}$ & 0 \\
\hline f̊ & 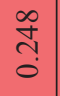 & $\widehat{\widehat{\delta}}$ & $\begin{array}{l}\vec{\infty} \\
\infty \\
0 \\
0\end{array}$ & 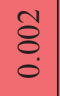 & ठ̊ & 0 & 0 & $\begin{array}{l}\hat{\delta} \\
\dot{i} \\
\dot{i}\end{array}$ & $\underset{\overbrace{}}{\tilde{y}}$ & 0 & 0 & $\stackrel{0}{\circ}$ & चֶ. & ठ̊. & ¿़े & 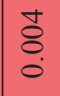 & 0 & 0 & 0 \\
\hline$\stackrel{\infty}{\infty}$ & $\stackrel{\oslash}{\cong}$ & \pm & $\begin{array}{l}\tilde{b} \\
+\end{array}$ & - & - & 0 & 0 & $\frac{\hat{a}}{\stackrel{n}{a}}$ & 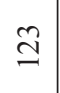 & 0 & 0 & $\underline{2}$ & $\hat{\sim}$ & - & $N$ & $\sim$ & 0 & 0 & 0 \\
\hline ठे. & $\begin{array}{l}0 \\
\circ \\
0\end{array}$ & $\begin{array}{l}\overline{8} \\
\dot{0}\end{array}$ & ố. & $\begin{array}{l}\overline{8} \\
\dot{0}\end{array}$ & 0 & 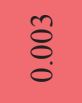 & 0 & 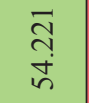 & $\begin{array}{l}\infty \\
\stackrel{0}{0} \\
0\end{array}$ & ठ̊. & $\begin{array}{l}n \\
\vdots \\
0 \\
0\end{array}$ & $\begin{array}{l}\text { त̂ } \\
\text { ठ். }\end{array}$ & $\begin{array}{l}\overline{8} \\
\dot{0}\end{array}$ & 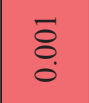 & 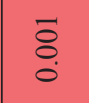 & $\overline{8}$ & 0 & $\vec{\circ}$ & $\begin{array}{l}\overline{8} \\
\dot{0}\end{array}$ \\
\hline i & $\stackrel{\sim}{\sim}$ & - & $\stackrel{\infty}{n}$ & $N$ & 0 & $\nabla$ & 0 & $\begin{array}{l}\tilde{N} \\
\infty \\
\infty\end{array}$ & 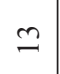 & $m$ & $\infty$ & $\stackrel{r}{r}$ & $N$ & - & - & - & 0 & - & - \\
\hline ठें & 亏ें & 亏ें & ڤें & ลें & ڤे & ลें & ڤें & ఏे & aे & à & 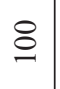 & ळ่ ठे & छे & $\stackrel{\circ}{\circ}$ & छे & ठें & ลें & ఏे & ลें \\
\hline 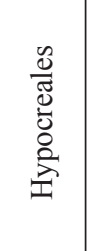 & 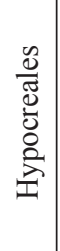 & 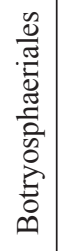 & 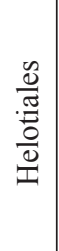 & 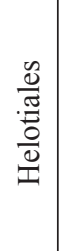 & 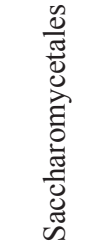 & 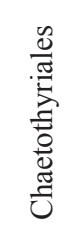 & 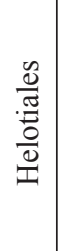 & 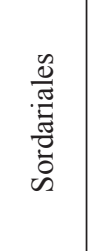 & 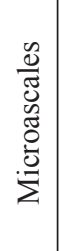 & 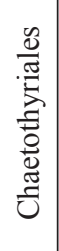 & 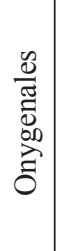 & 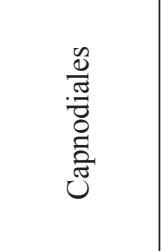 & 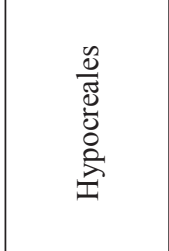 & $\begin{array}{l}\frac{0}{\sqrt{0}} \\
\frac{\pi}{0} \\
\frac{0}{0} \\
\frac{0}{2}\end{array}$ & 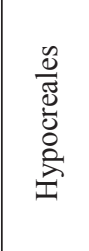 & 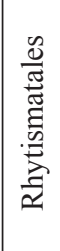 & 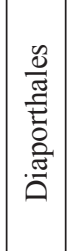 & 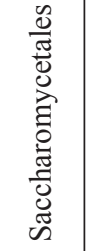 & $\begin{array}{l}\mathscr{0} \\
\frac{\mathbb{N}}{N} \\
\mathbb{N} \\
\Omega\end{array}$ \\
\hline 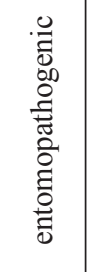 & 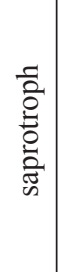 & 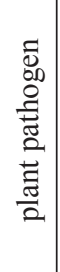 & 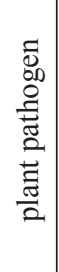 & 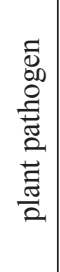 & 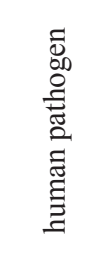 & 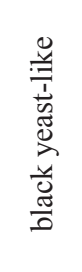 & 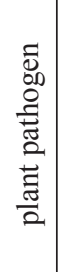 & 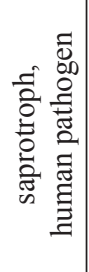 & 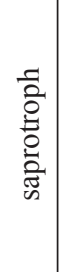 & & 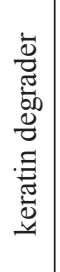 & 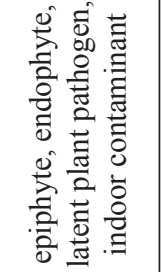 & 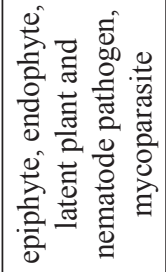 & 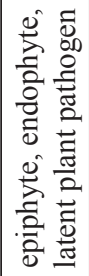 & 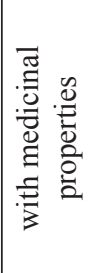 & 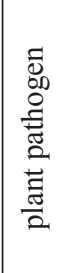 & 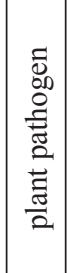 & 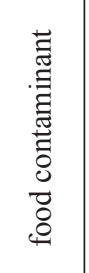 & 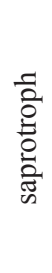 \\
\hline 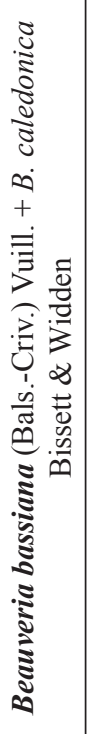 & 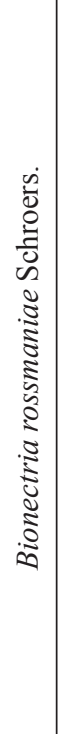 & 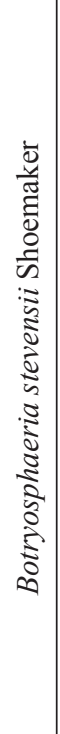 & 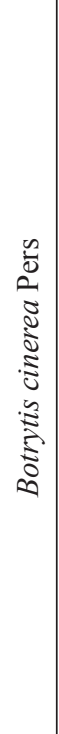 & 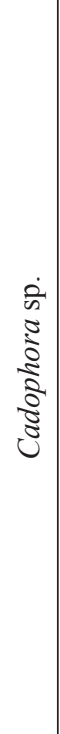 & 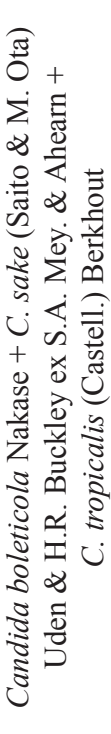 & 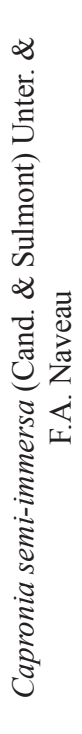 & 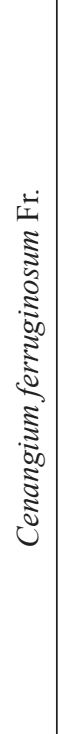 & 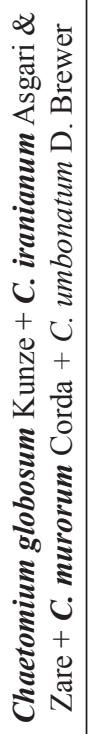 & 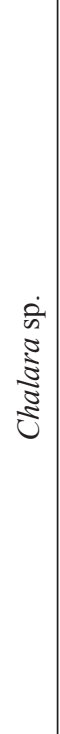 & 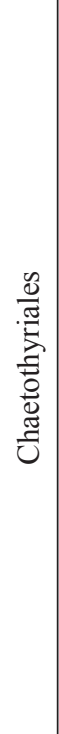 & 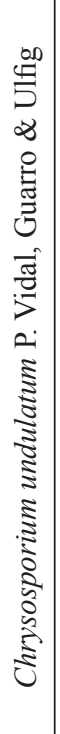 & 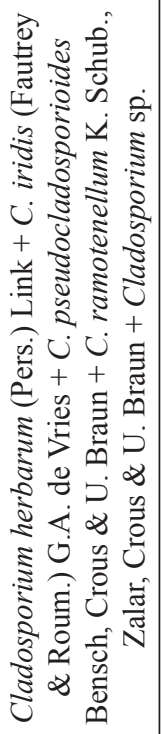 & 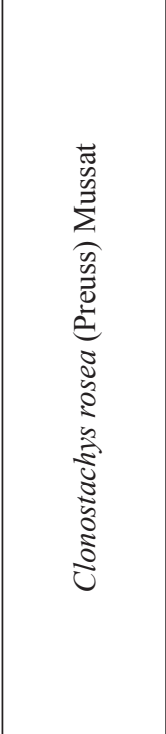 & 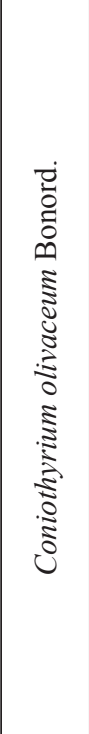 & 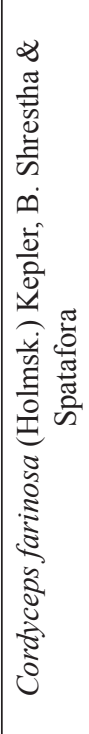 & 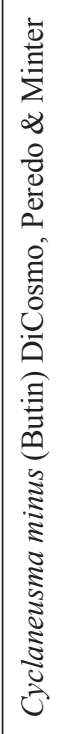 & 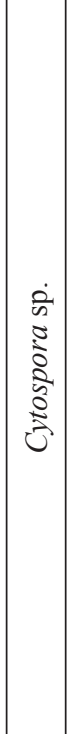 & 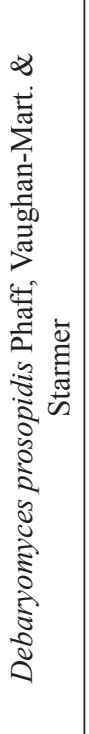 & 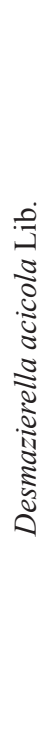 \\
\hline$\infty$ & $\sigma^{\circ}$ & $\dot{\ominus}$ & $\dot{=}$ & 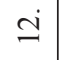 & $\dot{g}$ & \pm & $\because$ & $\stackrel{0}{0}$ & 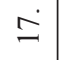 & $\stackrel{\infty}{\infty}$ & $\stackrel{\circ}{2}$ & $\dot{i}$ & $\vec{N}$ & ป่ & $\ddot{\lambda}$ & $\stackrel{\sim}{d}$ & $\ddot{\imath}$ & $\dot{\sim}$ & $\dot{\sim}$ \\
\hline
\end{tabular}




\begin{tabular}{|c|c|c|c|c|c|c|c|c|c|c|c|c|c|c|c|c|c|c|c|c|}
\hline $\begin{array}{l}n \\
\tilde{c} \\
0\end{array}$ & $\vec{n}$ & $\begin{array}{l}n \\
\delta \\
0\end{array}$ & $\begin{array}{c}\text { ळे } \\
\infty \\
0 \\
0\end{array}$ & $\begin{array}{l}1 \\
0 \\
0\end{array}$ & $\begin{array}{l}\hat{\infty} \\
\stackrel{0}{0}\end{array}$ & $\overrightarrow{\vec{n}}$ & 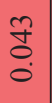 & 0 & $\begin{array}{l}0 \\
0 \\
0 \\
0\end{array}$ & $\stackrel{\Xi}{\circ}$ & तิ & $\begin{array}{l}\Xi \\
\\
0\end{array}$ & $\stackrel{\cong}{0}$ & 合 & 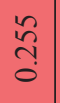 & $\stackrel{\infty}{=}$ & ஜ̊. & $\frac{8}{\circ}$ & 8 & : \\
\hline ป & $\overrightarrow{\widetilde{N}}$ & $\sim$ & 'f & $n$ & r & $\stackrel{\sim}{\sim}$ & $\stackrel{\infty}{\sim}$ & 0 & ले & $\sim$ & $a$ & fo & $i n$ & లే & 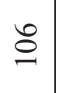 & qे & $\cong$ & $\stackrel{7}{2}$ & $m$ & \\
\hline 0 & तु & 0 & $\overrightarrow{5}$ & $\begin{array}{l}1 \\
\delta \\
0 \\
0\end{array}$ & 0 & $\begin{array}{l}\text { ते } \\
\text { గุ }\end{array}$ & 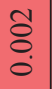 & 0 & 0 & 0 & 0 & $\begin{array}{l}\dot{0} \\
\dot{0} \\
0\end{array}$ & 0 & 0 & $\begin{array}{l}0 \\
0 \\
0 \\
0\end{array}$ & 0 & 0 & $\begin{array}{l}\mathscr{8} \\
\stackrel{0}{0} \\
0\end{array}$ & $\begin{array}{l}\stackrel{2}{O} \\
\frac{1}{0}\end{array}$ & \\
\hline 0 & $=$ & 0 & $\hat{n}$ & -1 & 0 & 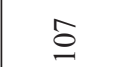 & - & 0 & 0 & 0 & 0 & $\sim$ & 0 & 0 & in & 0 & 0 & $m$ & in & \\
\hline$\overline{8}$ & 0 & 0 & $\begin{array}{l}\infty \\
\stackrel{8}{0} \\
0\end{array}$ & $\overrightarrow{8}$ & $\begin{array}{l}\text { กิ } \\
0 \\
0\end{array}$ & $\begin{array}{l}\stackrel{+}{0} \\
\stackrel{0}{0}\end{array}$ & 0 & $\begin{array}{l}0 \\
\Xi \\
0\end{array}$ & $\overrightarrow{8}$ & 0 & 0 & $\begin{array}{l}\overline{8} \\
\dot{0}\end{array}$ & 0 & $\frac{d}{0}$ & 0 & 0 & 0 & $\begin{array}{c}\tilde{8} \\
\dot{0}\end{array}$ & $\begin{array}{l}\stackrel{\circ}{\pi} \\
\text { o }\end{array}$ & \\
\hline- & 0 & 0 & 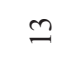 & $\sim$ & $\dot{m}$ & $n$ & 0 & 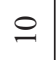 & - & 0 & 0 & - & 0 & 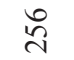 & 0 & 0 & 0 & in & సे & \\
\hline @̊̀ & ふें & ڤ̊ํ & छे̊ & 亏ั & ลे & छे & $\stackrel{\circ}{\circ}$ & @ें & 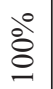 & ڤें & @ें & ڤें & ڤ̊ํㅇ & छे & ڤें & જ́ & ลे & ڤें & ڤ̆ & 8 \\
\hline $\begin{array}{l}\frac{u}{0} \\
\frac{0}{0} \\
\frac{0}{0} \\
\frac{0}{2}\end{array}$ & 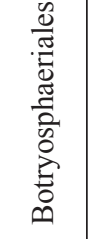 & & $\begin{array}{l}\frac{0}{0} \\
\frac{0}{\pi} \\
\frac{0}{00} \\
\frac{d}{2}\end{array}$ & 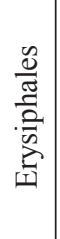 & 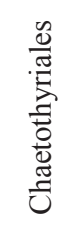 & 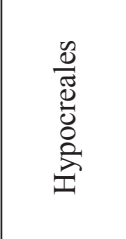 & 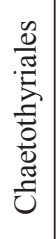 & 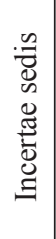 & 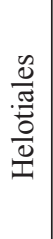 & 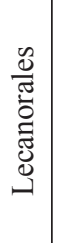 & 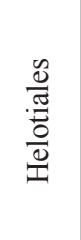 & & 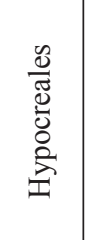 & 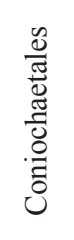 & & 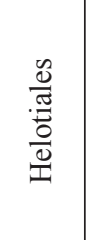 & 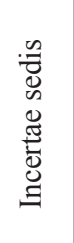 & 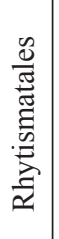 & 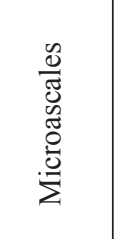 & $\frac{\mathscr{\vartheta}}{\pi}$ \\
\hline 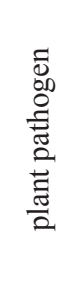 & 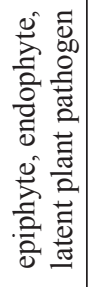 & & 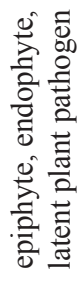 & 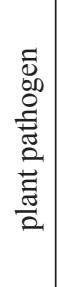 & 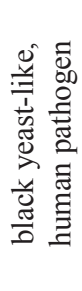 & 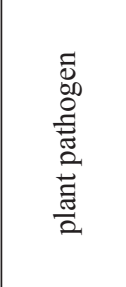 & & 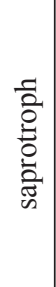 & 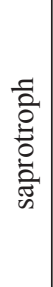 & 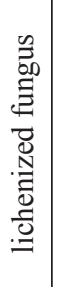 & 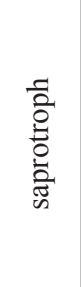 & 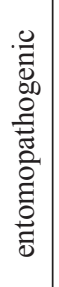 & 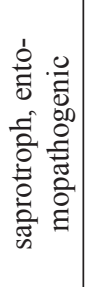 & 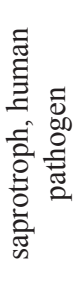 & & 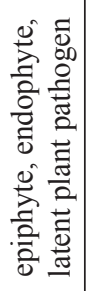 & 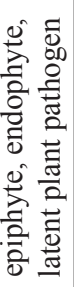 & 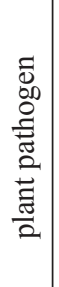 & 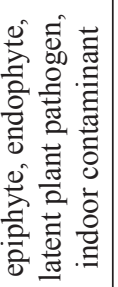 & డీ \\
\hline 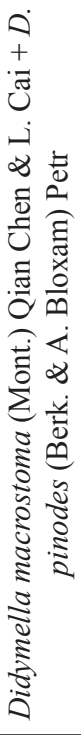 & 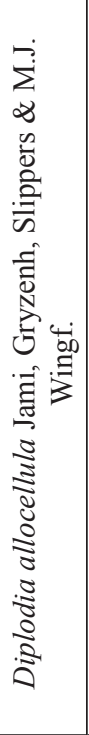 & 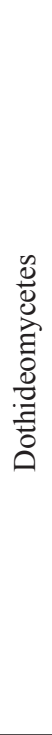 & 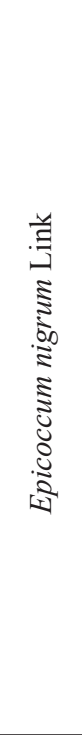 & 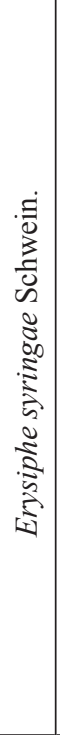 & 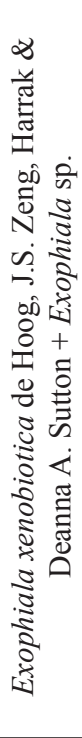 & 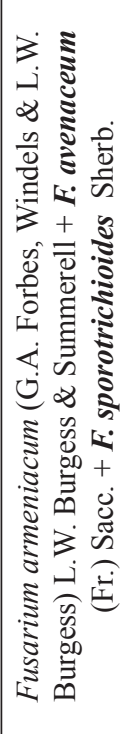 & 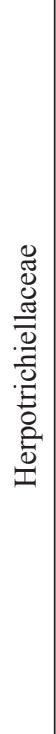 & 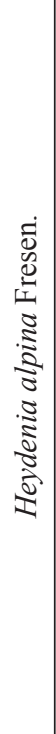 & 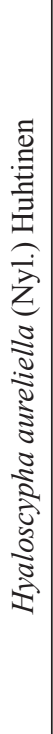 & 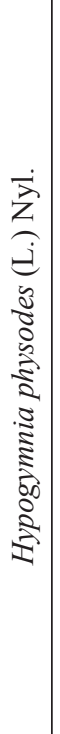 & 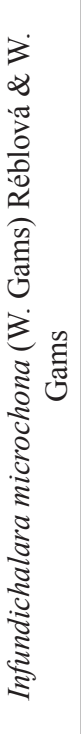 & 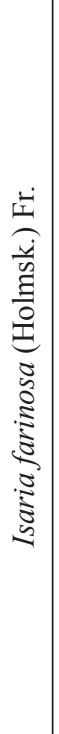 & 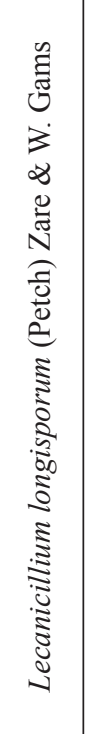 & 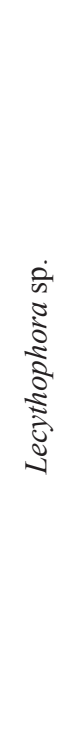 & 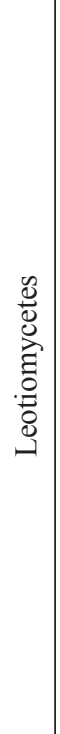 & 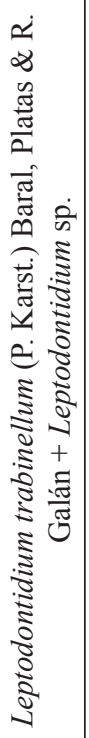 & 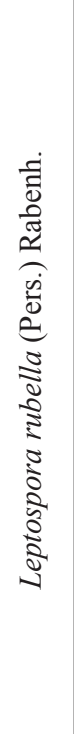 & 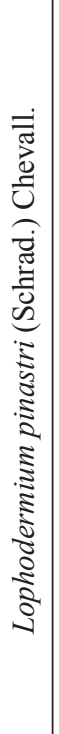 & 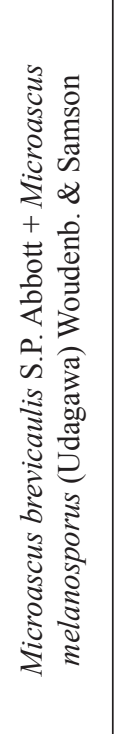 & 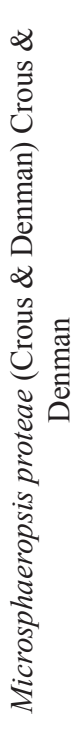 \\
\hline$\stackrel{\infty}{\sim}$ & $\stackrel{\lambda}{\dot{\lambda}}$ & లn & $\vec{m}$ & m & $m$ & $\bar{m}$ & $m$ & $m$ & $m$ & $\begin{array}{c}\dot{\infty} \\
m\end{array}$ & $\dot{m}$ & $\dot{f}$ & $\dot{\vec{\gamma}}$ & ช่ & $\dot{\vartheta}$ & $\dot{f}$ & $\dot{q}$ & $\dot{b}$ & $\dot{f}$ & 䎹 \\
\hline
\end{tabular}




\begin{tabular}{|c|c|c|c|c|c|c|c|c|c|c|c|c|c|c|c|c|c|c|}
\hline 0 & $\begin{array}{l}\vec{b} \\
\overrightarrow{0}\end{array}$ & $\begin{array}{l}\tilde{O} \\
\text { Oे } \\
0\end{array}$ & $\begin{array}{l}\text { m} \\
0 \\
0\end{array}$ & 0 & ¿্. & $\begin{array}{l}\tilde{\delta} \\
0\end{array}$ & $\begin{array}{l}1 \\
\dot{8} \\
0\end{array}$ & 웅. & 0 & $\begin{array}{l}n \\
m \\
0\end{array}$ & ठे. & $\tilde{n}_{-}^{n}$ & 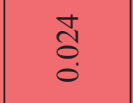 & $\stackrel{5}{0}$ & $\begin{array}{l}\tilde{\delta} \\
0 \\
0\end{array}$ & $\begin{array}{l}\tilde{\delta} \\
0 \\
0\end{array}$ & 0 & 0 \\
\hline 0 & $\overline{6}$ & $a$ & $\stackrel{\infty}{-}$ & 0 & $\Xi$ & - & $m$ & $\stackrel{2}{ }$ & 0 & in & $\simeq$ & के & $\stackrel{ }{ }$ & $r$ & $a$ & $a$ & 0 & 0 \\
\hline 0 & 0 & 0 & $\begin{array}{l}0 \\
\stackrel{0}{0} \\
0\end{array}$ & 0 & 0 & 0 & 0 & 0 & 0 & $\stackrel{0}{0}$ & 0 & 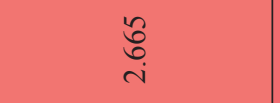 & 0 & 0 & 0 & 0 & 0 & 0 \\
\hline 0 & 0 & 0 & - & 0 & 0 & 0 & 0 & 0 & 0 & in & 0 & $\stackrel{\infty}{\stackrel{一}{్}}$ & 0 & 0 & 0 & 0 & 0 & 0 \\
\hline$\overline{8}$ & $\begin{array}{l}\tilde{\partial} \\
\dot{0}\end{array}$ & 0 & 0 & $\begin{array}{l}\infty \\
\stackrel{0}{0} \\
0\end{array}$ & 0 & 0 & 0 & 0 & $\overline{8}$ & 0 & 0 & $\begin{array}{l}\frac{J}{2} \\
\stackrel{a}{a}\end{array}$ & 0 & 0 & 0 & 0 & $\overline{8}$ & $\begin{array}{l}\text { ¿े } \\
\stackrel{0}{0}\end{array}$ \\
\hline- & $\stackrel{i}{\sim}$ & 0 & 0 & 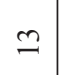 & 0 & 0 & 0 & 0 & $N$ & 0 & 0 & 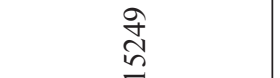 & 0 & 0 & 0 & 0 & - & 0 \\
\hline ڤे & aें & ลे & ลें & ลें & ڤั & ठें & å & ڤ̊ & ڤ̊ํㅇ & 㝘 & ลे & જ่ & @̊̀ & $\begin{array}{l}\circ \\
\infty \\
\sigma\end{array}$ & ○ें & @̊े & @े & @े \\
\hline 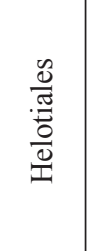 & 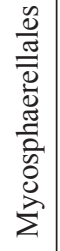 & 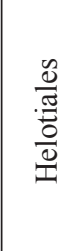 & 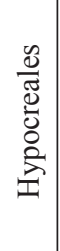 & $\begin{array}{l}\frac{0}{0} \\
\frac{0}{\pi} \\
\frac{0}{0} \\
\frac{0}{02} \\
\frac{d}{2}\end{array}$ & 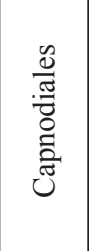 & 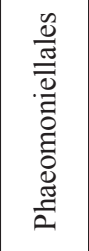 & 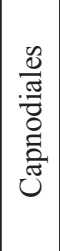 & 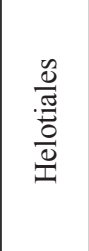 & 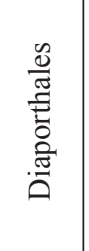 & 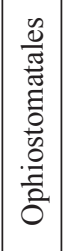 & $\begin{array}{l}\frac{0}{n} \\
\frac{\pi}{0} \\
\frac{0}{0} \\
\frac{0}{2}\end{array}$ & 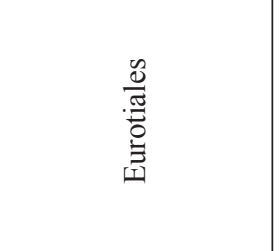 & 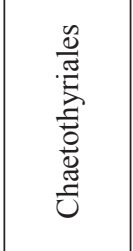 & 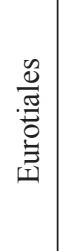 & 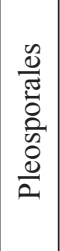 & 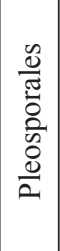 & $\frac{\frac{\tilde{d}}{\vec{\pi}}}{\frac{\vec{\pi}}{\vec{x}}}$ & 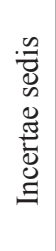 \\
\hline 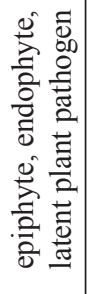 & 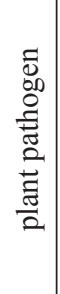 & 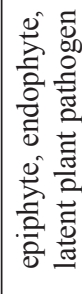 & 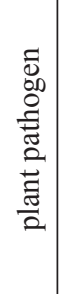 & 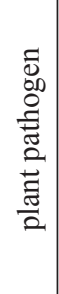 & 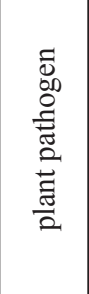 & 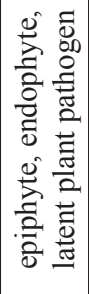 & 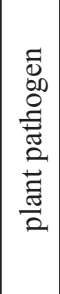 & 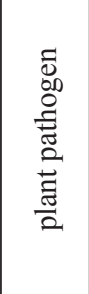 & 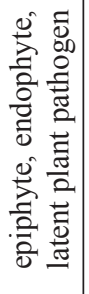 & 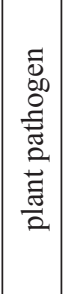 & 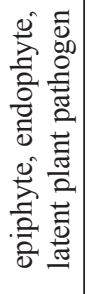 & 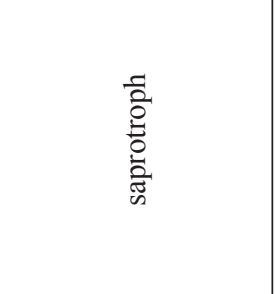 & 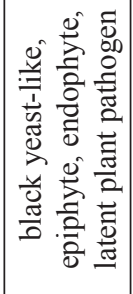 & 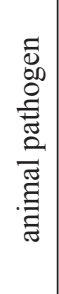 & 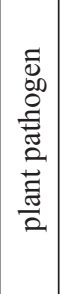 & & 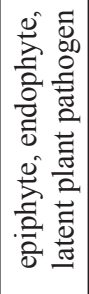 & 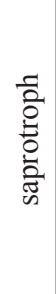 \\
\hline 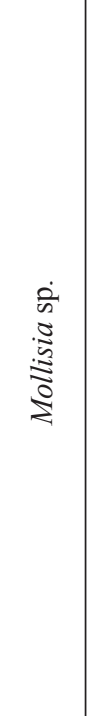 & 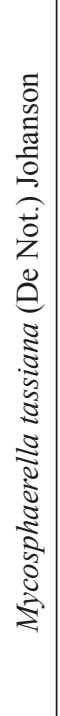 & 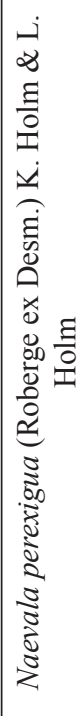 & 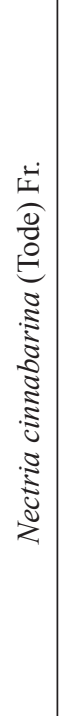 & 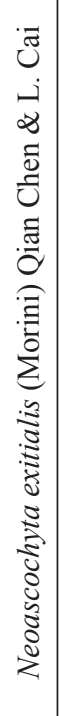 & 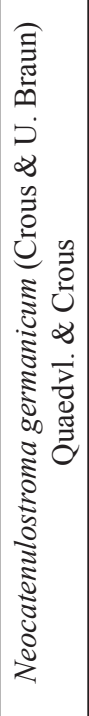 & 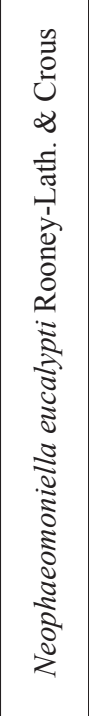 & 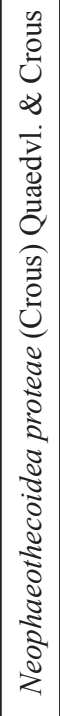 & 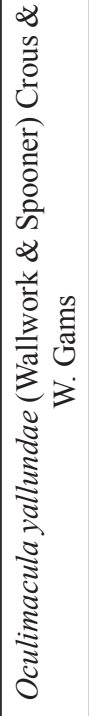 & 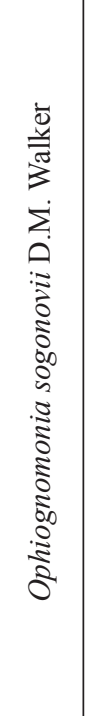 & 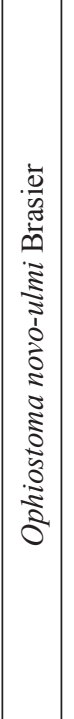 & 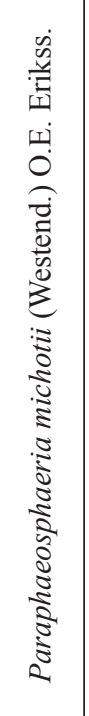 & 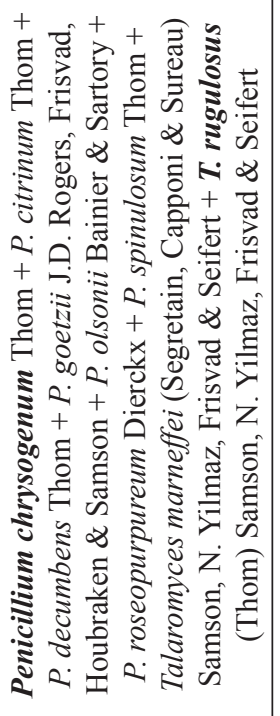 & 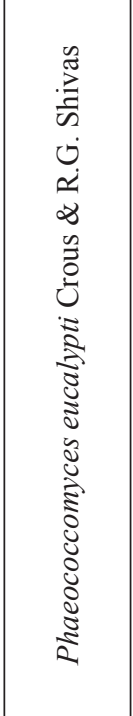 & 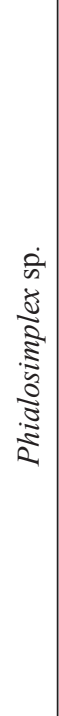 & 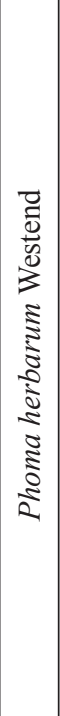 & 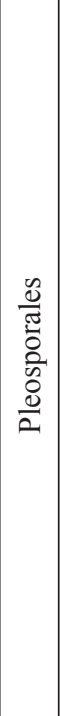 & 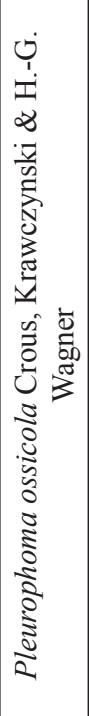 & 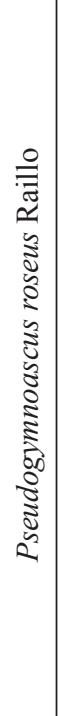 \\
\hline$\dot{q}$ & $\dot{n}$ & $\vec{n}$ & in & $\dot{n}$ & $\stackrel{\dot{n}}{ }$ & in & $\ddot{n}$ & in & $\stackrel{\infty}{n}$ & in & $\dot{8}$ & $\dot{\sigma}$ & ชิ่ & ல் & $\dot{b}$ & 8 & $\ddot{8}$ & $\dot{6}$ \\
\hline
\end{tabular}




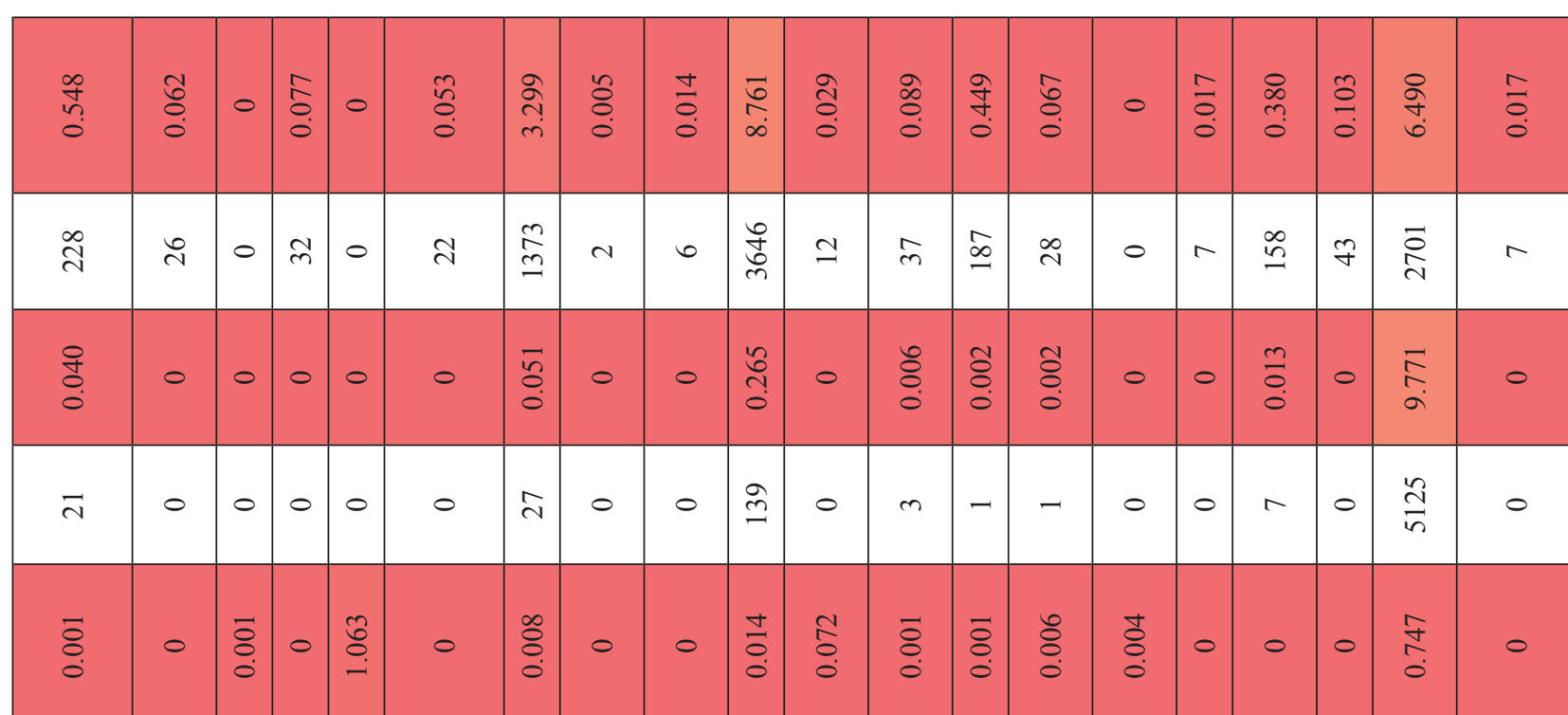

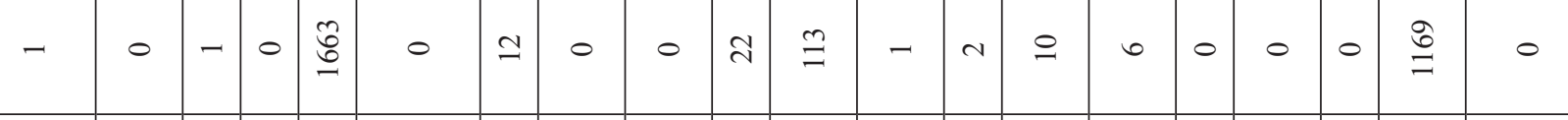

ఏे ठे

\begin{tabular}{|c|c|c|c|c|c|c|c|c|c|c|c|c|c|c|c|c|c|c|}
\hline $\begin{array}{l}\frac{0}{0} \\
\frac{\pi}{0} \\
\frac{0}{0} \\
0 \\
\frac{0}{2}\end{array}$ & 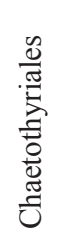 & 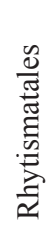 & 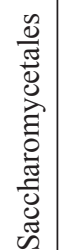 & 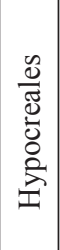 & 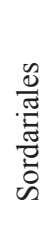 & 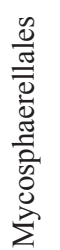 & 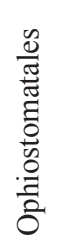 & 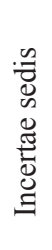 & 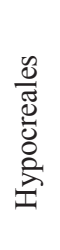 & 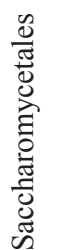 & 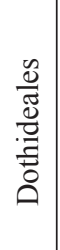 & 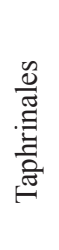 & 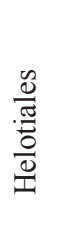 & $\begin{array}{l}\frac{0}{0} \\
\frac{\pi}{0} \\
\frac{0}{0} \\
\frac{0}{0} \\
\equiv\end{array}$ & 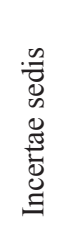 & 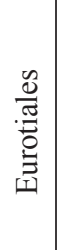 & 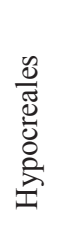 & 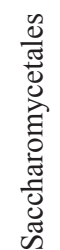 \\
\hline
\end{tabular}

\begin{tabular}{|c|c|c|c|c|c|c|c|c|c|c|c|c|c|c|c|c|c|}
\hline 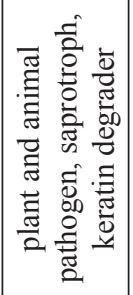 & 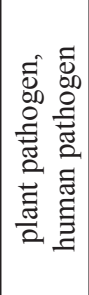 & 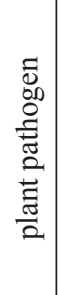 & 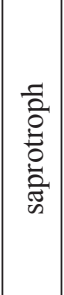 & 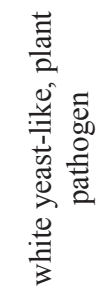 & 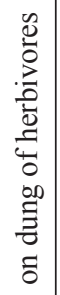 & 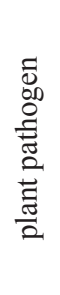 & 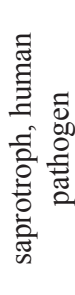 & 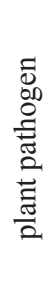 & 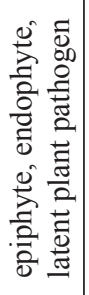 & 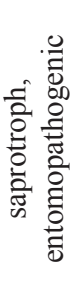 & 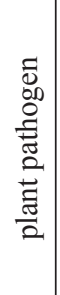 & 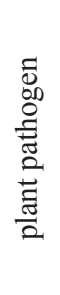 & 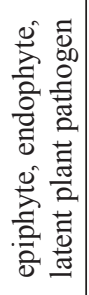 & 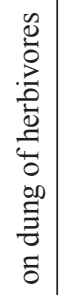 & 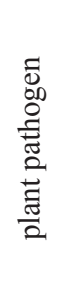 & & 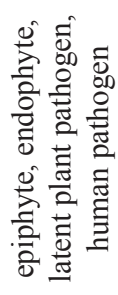 \\
\hline
\end{tabular}

\begin{tabular}{|c|c|c|c|c|c|c|c|c|c|c|c|c|c|c|c|c|c|c|c|}
\hline 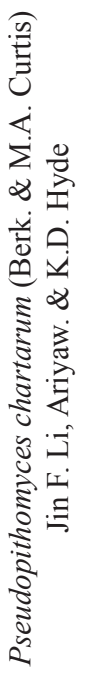 & 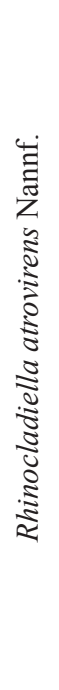 & 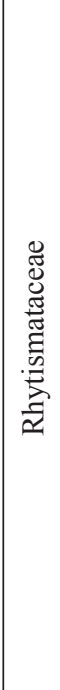 & 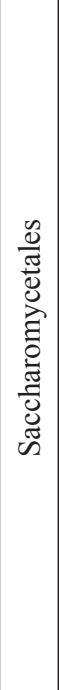 & 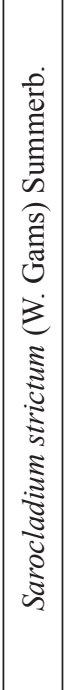 & 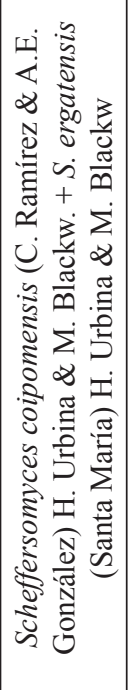 & 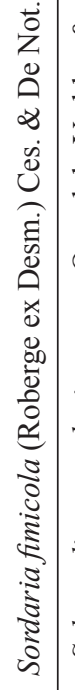 & 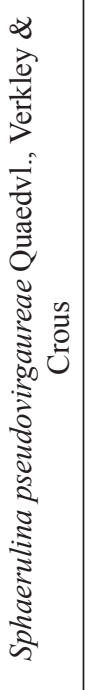 & 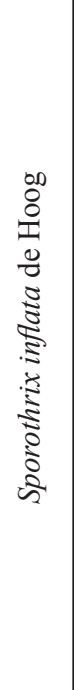 & 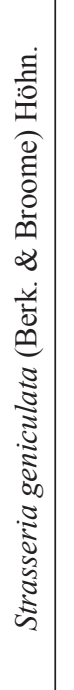 & 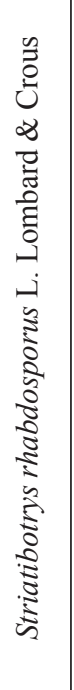 & 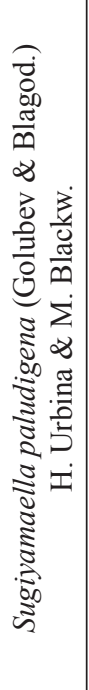 & 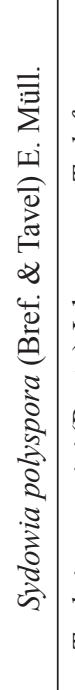 & 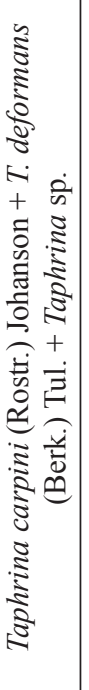 & 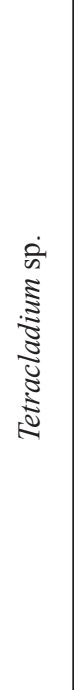 & 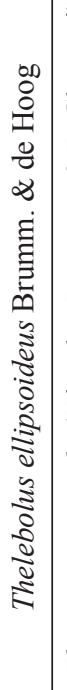 & 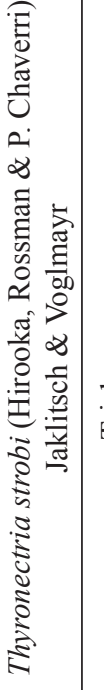 & 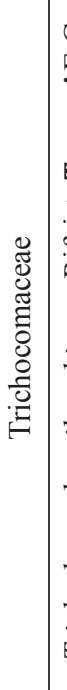 & 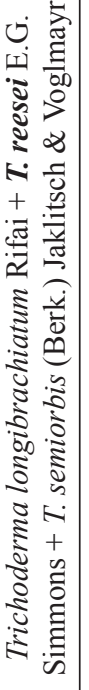 & 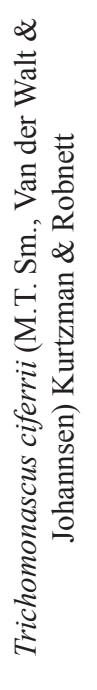 \\
\hline$\stackrel{\infty}{\infty}$ & 80 & $\dot{R}$ & $\dot{R}$ & i & $\stackrel{2}{2}$ & $\stackrel{\mathbb{Z}}{ }$ & $\stackrel{n}{i}$ & $\stackrel{0}{\circ}$ & $\stackrel{\circ}{\therefore}$ & $\stackrel{\infty}{\curvearrowright}$ & $2^{\circ}$ & $\dot{\infty}$ & $\dot{\infty}$ & ஸ் & $\infty$ & $+\infty$ & $\infty$ & $\dot{\infty}$ & 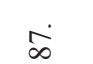 \\
\hline
\end{tabular}




\begin{tabular}{|c|c|c|c|c|c|c|c|c|c|c|c|c|c|c|c|c|c|c|c|c|c|}
\hline $\begin{array}{l}\infty \\
\tilde{\delta} \\
0 \\
0\end{array}$ & $\begin{array}{l}0 \\
\text { రై } \\
0\end{array}$ & $\begin{array}{l}\stackrel{0}{\hat{\infty}} \\
\stackrel{=}{=}\end{array}$ & $\begin{array}{l}\dot{o} \\
\dot{0} \\
\dot{0}\end{array}$ & $\frac{\overline{0}}{0}$ & 0 & గ్ & 0 & 0 & $\stackrel{-}{\circ}$ & $\stackrel{\infty}{=}$ & 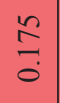 & 0 & $\frac{8}{\circ}$ & $\begin{array}{l}0 \\
\stackrel{0}{0} \\
0\end{array}$ & 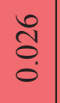 & 0 & $\begin{array}{l}0 \\
0 \\
0 \\
0\end{array}$ & $\frac{8}{0}$ & $\begin{array}{l}\infty \\
\approx \\
0 \\
0\end{array}$ & $\begin{array}{l}\stackrel{+}{0} \\
\stackrel{0}{0} \\
0\end{array}$ & $\stackrel{0}{0}$ \\
\hline$\stackrel{\circ}{-}$ & $=$ & 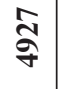 & $\cong$ & F & 0 & 䄵 & 0 & 0 & in & $\vec{q}$ & $\approx$ & 0 & $\stackrel{2}{2}$ & $=$ & $=$ & 0 & $\vec{v}$ & $\Varangle$ & $\bar{F}$ & $m$ & $r$ \\
\hline 0 & 0 & ثิ: & $\begin{array}{l}\infty \\
\stackrel{0}{0} \\
0\end{array}$ & 0 & 0 & $\begin{array}{l}m \\
0 \\
0\end{array}$ & 0 & 0 & 0 & 0 & $\left|\begin{array}{c}\vec{\delta} \\
0 \\
0\end{array}\right|$ & 0 & $\begin{array}{l}0 \\
0 \\
0 \\
0\end{array}$ & 0 & 0 & 0 & 0 & $\bar{\sigma}$ & 0 & 0 & 0 \\
\hline 0 & 0 & 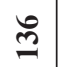 & $\nabla$ & 0 & 0 & $r$ & 0 & 0 & 0 & 0 & $=$ & 0 & in & 0 & 0 & 0 & 0 & 0 & 0 & 0 & 0 \\
\hline $\overrightarrow{8}$ & 0 & $\stackrel{\infty}{\stackrel{\infty}{0}} \stackrel{0}{\stackrel{\infty}{0}}$ & $\begin{array}{l}\overline{8} \\
\dot{0}\end{array}$ & $\overrightarrow{8}$ & $\begin{array}{l}0 \\
\dot{8} \\
0\end{array}$ & $\begin{array}{l}\tilde{\Xi} \\
\text { ठ் }\end{array}$ & $\overline{8}$ & $\overline{8}$ & 0 & 0 & $\begin{array}{l}\hat{y} \\
0 \\
0\end{array}$ & $\begin{array}{l}\tilde{8} \\
\delta \\
\delta\end{array}$ & $\begin{array}{l}\tilde{8} \\
\dot{0}\end{array}$ & 0 & 0 & $\begin{array}{l}0 \\
0 \\
0\end{array}$ & $\stackrel{\text { }}{\stackrel{0}{0}}$ & $\begin{array}{l}\tilde{\delta} \\
\delta\end{array}$ & 0 & $\stackrel{8}{8}$ & 0 \\
\hline$\sim$ & 0 & $\stackrel{\mathscr{0}}{\stackrel{\Xi}{\Xi}}$ & - & - & \pm & $\dot{m}$ & $N$ & $N$ & 0 & 0 & $\sigma$ & $n$ & $\nabla$ & 0 & 0 & $a$ & $\bar{n}$ & in & 0 & N & 0 \\
\hline aे & ลें & & $\stackrel{\circ}{\circ}$ & $\stackrel{\circ}{0}$ & ลें & ลें & ลें & ล̊ & $\stackrel{\circ}{0}$ & $\stackrel{\circ}{\circ}$ & ลें & ఏे & $\stackrel{\circ}{0}$ & ลें & aे & ఏें & á & ఏें & ळे & ò & ลें \\
\hline 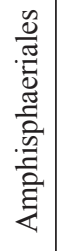 & 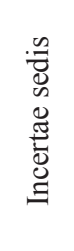 & & 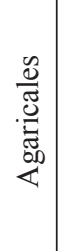 & 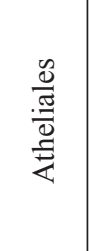 & 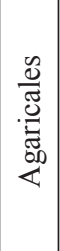 & 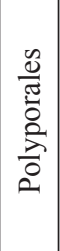 & 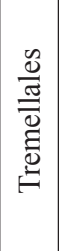 & 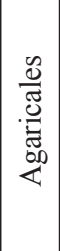 & 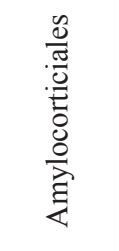 & 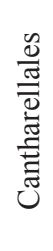 & 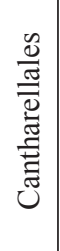 & 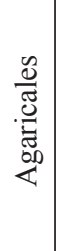 & 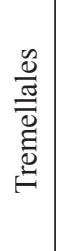 & 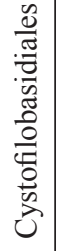 & 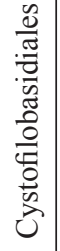 & $\begin{array}{l}\frac{0}{0} \\
\frac{0}{\pi} \\
0 \\
\frac{2}{2} \\
\frac{0}{0} \\
2\end{array}$ & 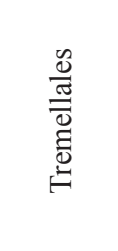 & 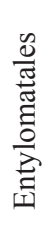 & 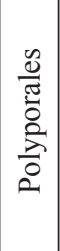 & 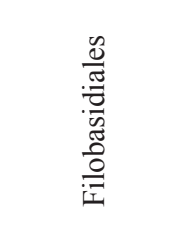 & 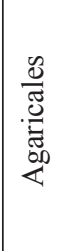 \\
\hline 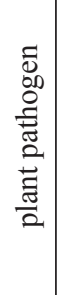 & 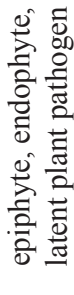 & & 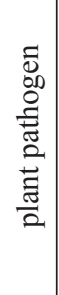 & 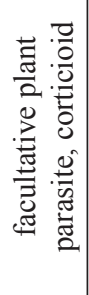 & 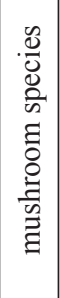 & 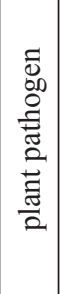 & 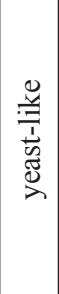 & 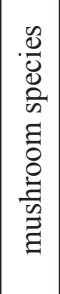 & 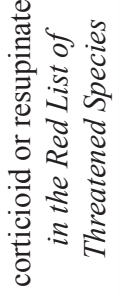 & & 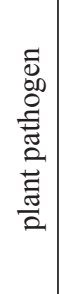 & 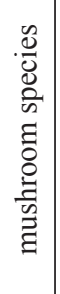 & 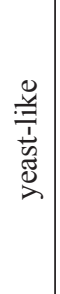 & 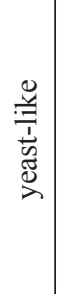 & 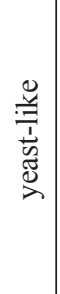 & 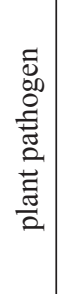 & 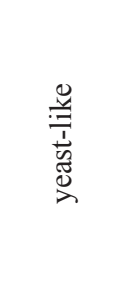 & & 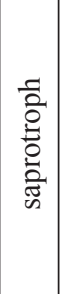 & 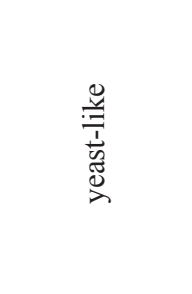 & 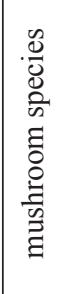 \\
\hline 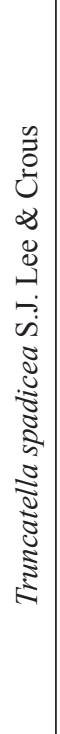 & 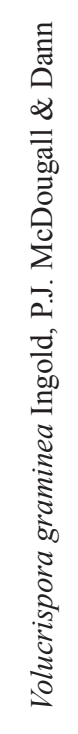 & 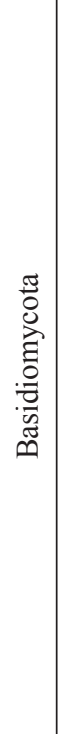 & 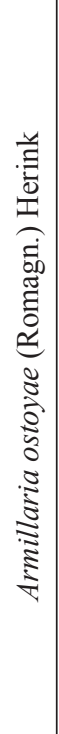 & 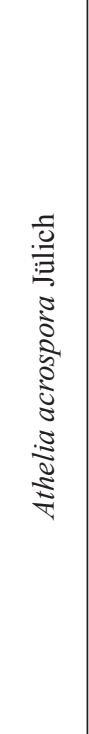 & 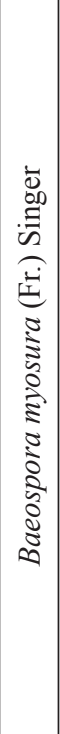 & 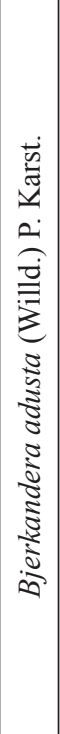 & 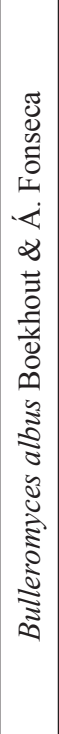 & 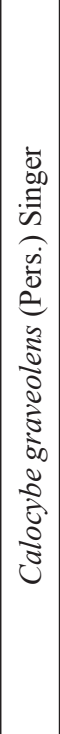 & 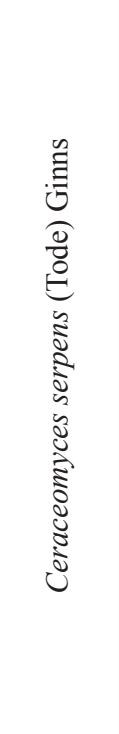 & 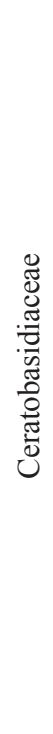 & 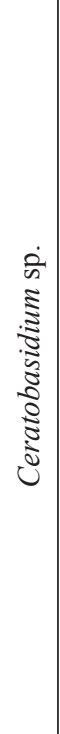 & 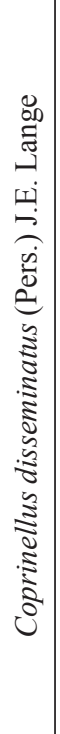 & 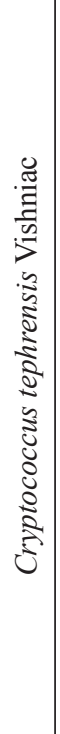 & 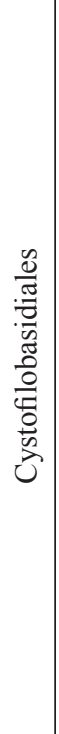 & 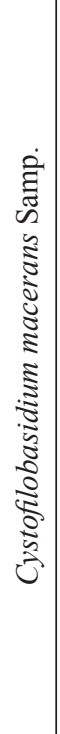 & 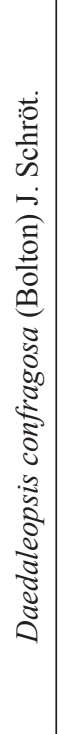 & 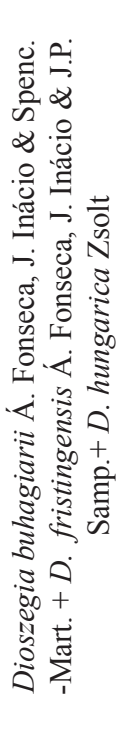 & 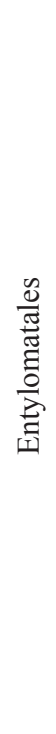 & 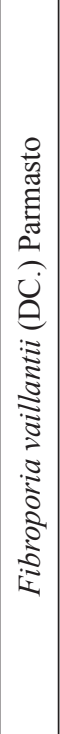 & 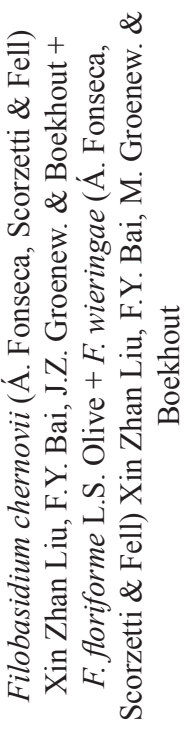 & 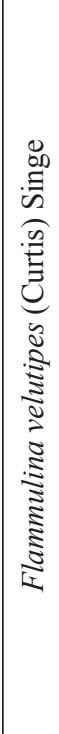 \\
\hline$\dot{\infty}$ & $\infty$ & & -7 & $i$ & $\dot{n}$ & $\dot{r}$ & $i n$ & 0 & $\therefore$ & $\infty$ & $\sigma^{\circ}$ & $\dot{\ominus}$ & $\dot{=}$ & $\stackrel{\sim}{\simeq}$ & $\dot{\sim}$ & $\dot{ \pm}$ & in & $\stackrel{\dot{\varphi}}{ }$ & $\stackrel{\check{1}}{0}$ & $\stackrel{\infty}{\mathscr{D}}$ & $\stackrel{2}{2}$ \\
\hline
\end{tabular}




\begin{tabular}{|c|c|c|c|c|c|c|c|c|c|c|c|c|c|c|c|c|c|c|c|c|}
\hline đ̊. & \begin{tabular}{l} 
in \\
\multirow{2}{c}{}
\end{tabular} & 0 & $\begin{array}{l}\text { ț } \\
\text { Oे }\end{array}$ & 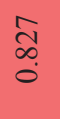 & $\begin{array}{l}\text { ठे. } \\
\text { ¿े }\end{array}$ & $\begin{array}{l}\infty \\
\tilde{o} \\
0 \\
0\end{array}$ & 0 & $\begin{array}{l}\stackrel{t}{n} \\
\text { ñ }\end{array}$ & ले & $\frac{\cong}{0}$ & $\stackrel{\infty}{\stackrel{\circ}{0}}$ & 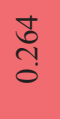 & $\vec{\delta}$ & $\begin{array}{l}\vec{\sigma} \\
\overrightarrow{0}\end{array}$ & 0 & $\underset{\delta}{\tilde{O}}$ & $\hat{\delta}$ & $\begin{array}{l}n \\
\delta \\
\delta \\
0\end{array}$ & $\begin{array}{l}\stackrel{+}{\circ} \\
\stackrel{-}{-}\end{array}$ & $\frac{\tilde{I}}{0}$ \\
\hline$\stackrel{\infty}{-}$ & ฮ̊ & 0 & 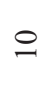 & $\underset{m}{\mathbb{f}}$ & $\simeq$ & 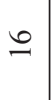 & 0 & $\tilde{\stackrel{\varrho}{\varrho}}$ & $\hat{n}$ & in & 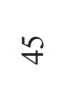 & $\stackrel{\ominus}{\Xi}$ & 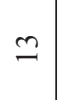 & $\hat{\sigma}$ & 0 & $\stackrel{\infty}{-}$ & $m$ & $\tilde{\lambda}$ & $\vec{s}$ & $\bar{n}$ \\
\hline 0 & 0 & 0 & 0 & 0 & 0 & 0 & 0 & $\begin{array}{l}\stackrel{0}{0} \\
\stackrel{0}{0}\end{array}$ & 0 & 0 & $\begin{array}{l}\text { to } \\
0 \\
0\end{array}$ & $\stackrel{\infty}{\circ}$ & 0 & $\begin{array}{l}\stackrel{m}{a} \\
\dot{0}\end{array}$ & 0 & 0 & 0 & 0 & $\begin{array}{l}\bar{n} \\
0 \\
0\end{array}$ & 0 \\
\hline 0 & 0 & 0 & 0 & 0 & 0 & 0 & 0 & $\circ$ & 0 & 0 & $\sim$ & $\nabla$ & 0 & $r$ & 0 & 0 & 0 & 0 & $\hat{\sim}$ & 0 \\
\hline 0 & $\begin{array}{l}\overrightarrow{8} \\
\dot{0}\end{array}$ & $\begin{array}{l}n \\
\stackrel{0}{0}\end{array}$ & 0 & $\stackrel{n}{\tilde{0}}$ & 0 & 0 & $\begin{array}{l}\tilde{O} \\
0 \\
0\end{array}$ & ธี & 0 & $\begin{array}{l}t \\
\stackrel{0}{0} \\
0\end{array}$ & $\begin{array}{l}\infty \\
\stackrel{0}{0} \\
0\end{array}$ & $\stackrel{\circ}{\circ}$ & 0 & $\begin{array}{l}\overrightarrow{8} \\
\stackrel{0}{0}\end{array}$ & \&̊ & 0 & $\overline{8}$ & 0 & $\begin{array}{l}\Delta \\
0 \\
0 \\
0\end{array}$ & 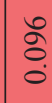 \\
\hline
\end{tabular}

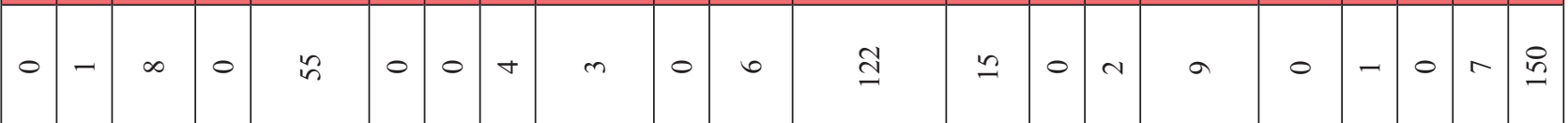

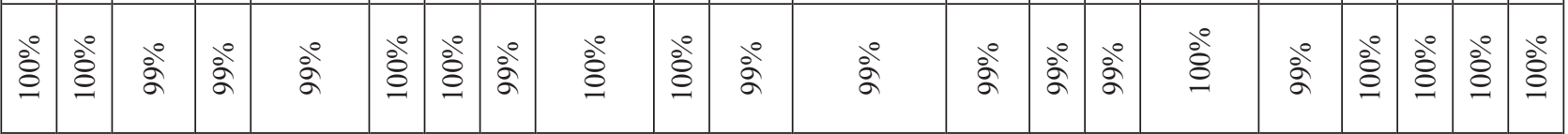

\begin{tabular}{|c|c|c|c|c|c|c|c|c|c|c|c|c|c|c|c|c|c|c|c|}
\hline & 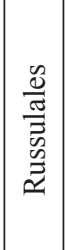 & 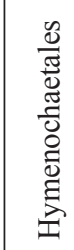 & 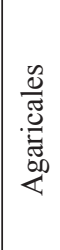 & 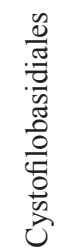 & 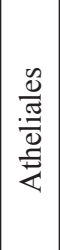 & 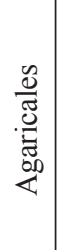 & 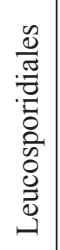 & 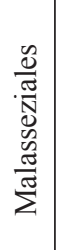 & $\begin{array}{l}\frac{n}{0} \\
\frac{0}{\pi} \\
\frac{0}{2} \\
\frac{2}{0} \\
0\end{array}$ & 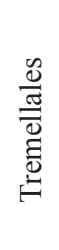 & 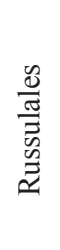 & $\begin{array}{l}\frac{0}{0} \\
\frac{0}{\pi} \\
\overline{:} \\
0 \\
0 \\
0\end{array}$ & 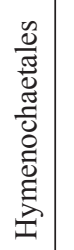 & $\begin{array}{l}\frac{v}{\pi} \\
\frac{0}{0} \\
\frac{0}{0} \\
0\end{array}$ & $\begin{array}{l}\frac{0}{0} \\
\frac{0}{\pi} \\
0 \\
\frac{0}{0} \\
0\end{array}$ & $\begin{array}{l}\frac{0}{0} \\
\frac{0}{5} \\
\frac{0}{0} \\
0 \\
0\end{array}$ & 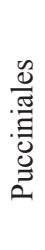 & 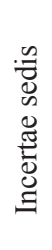 & 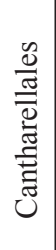 \\
\hline
\end{tabular}

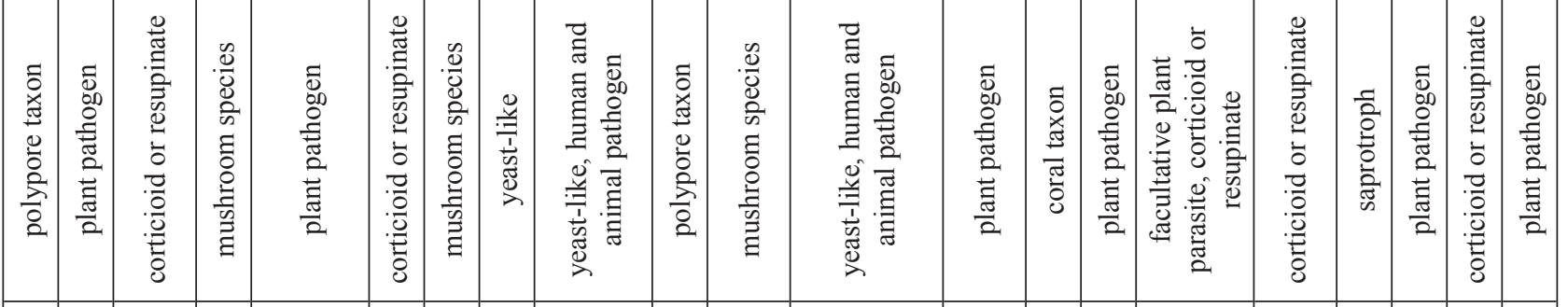

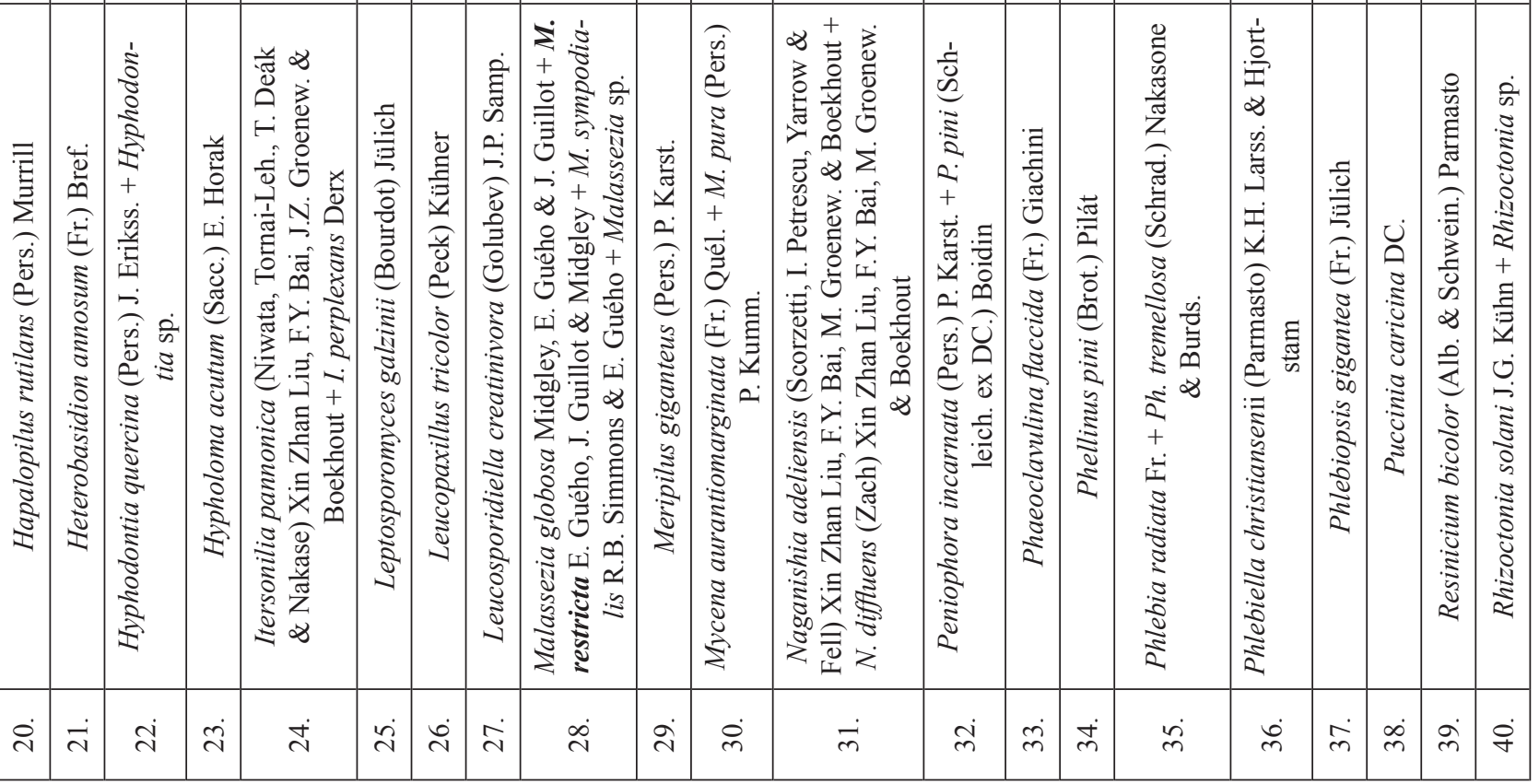




\begin{tabular}{|c|c|c|c|c|c|c|c|c|c|c|c|c|c|c|c|c|c|c|}
\hline 0 & 0 & $\begin{array}{l}\stackrel{m}{0} \\
\stackrel{0}{0}\end{array}$ & 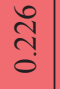 & 0 & $\frac{\mathcal{I}}{\stackrel{0}{0}}$ & $\begin{array}{l}\vec{F} \\
\dot{0}\end{array}$ & Ê. & 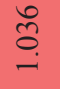 & $\begin{array}{l}\widetilde{1} \\
\text { ô. }\end{array}$ & $\begin{array}{l}\overline{\tilde{\sigma}} \\
\dot{0}\end{array}$ & $\begin{array}{l}0 \\
\tilde{\delta} \\
\stackrel{0}{0}\end{array}$ & 0 & $\begin{array}{c}\stackrel{i}{I} \\
0\end{array}$ & $\begin{array}{l}n \\
2 \\
n \\
o\end{array}$ & $\begin{array}{l}\text { I̊ } \\
\text { o. }\end{array}$ & 0 & $\stackrel{\infty}{\stackrel{\infty}{f}}$ & $\frac{m}{\square}$ \\
\hline 0 & 0 & $\stackrel{\infty}{\sim}$ & $t$ & 0 & in & 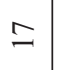 & in & $\bar{q}$ & $a$ & $\cong$ & $\because$ & 0 & $n$ & $\stackrel{\circ}{\vec{v}}$ & $=$ & 0 & $\stackrel{\partial}{ }$ & $\mathbb{N}$ \\
\hline 0 & 0 & $\begin{array}{l}1 \\
\tilde{0} \\
0\end{array}$ & $\begin{array}{l}\infty \\
\stackrel{0}{0} \\
0\end{array}$ & 0 & 0 & 0 & $\begin{array}{l}0 \\
\stackrel{0}{0} \\
\stackrel{0}{0}\end{array}$ & $\begin{array}{l}8 \\
8 \\
0\end{array}$ & 0 & 0 & 0 & 0 & 0 & $\begin{array}{l}0 \\
\tilde{0} \\
0 \\
0\end{array}$ & 0 & 0 & 0 & $\begin{array}{l}\dot{0} \\
8 \\
0\end{array}$ \\
\hline 0 & 0 & - & 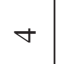 & 0 & 0 & 0 & $\stackrel{J}{\sim}$ & $m$ & 0 & 0 & 0 & 0 & 0 & $\curvearrowright$ & 0 & 0 & 0 & $v$ \\
\hline $\begin{array}{l}8 \\
\stackrel{0}{0}\end{array}$ & \&े. & $\frac{1}{\tilde{O}}$ & $\begin{array}{l}0 \\
\tilde{8} \\
0\end{array}$ & $\begin{array}{l}0 \\
\tilde{8} \\
0\end{array}$ & 0 & $\overrightarrow{8}$ & 0 & 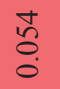 & $\begin{array}{l}\tilde{8} \\
\dot{0}\end{array}$ & 0 & $\tilde{\delta}$ & $\begin{array}{l}0 \\
\tilde{8}\end{array}$ & $\begin{array}{l}0 \\
\delta \\
0\end{array}$ & 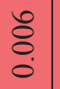 & $\overline{8}$ & $\hat{\tilde{\delta}}$ & $\vec{\circ}$ & $\begin{array}{l}0 \\
\tilde{8} \\
0 \\
0\end{array}$ \\
\hline 으 & ઇ & $\bar{m}$ & $\nabla$ & in & 0 & $\sim$ & 0 & $\mathscr{\infty}$ & $\nabla$ & 0 & in & in & $\circ$ & 으 & - & $\stackrel{\infty}{n}$ & - & $\infty$ \\
\hline ลें & ठ̊े & ఏे & ठ̊ำ & ठ̊ํ․ & $\stackrel{\circ}{\circ}$ & ڤें & ठे & ळे & ळे & $\stackrel{\circ}{\circ}$ & ठั๋ & ळे & ळे & ڤें & م่ & ลे & $\stackrel{\circ}{\circ}$ & ลे \\
\hline 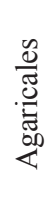 & 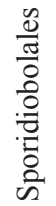 & $\begin{array}{l}\frac{0}{0} \\
\frac{0}{0} \\
\frac{0}{0} \\
\frac{0}{0} \\
\frac{0}{0} \\
\text { की }\end{array}$ & 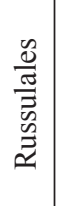 & 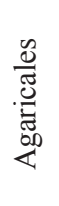 & $\begin{array}{l}\frac{0}{0} \\
\frac{0}{\pi} \\
\frac{\pi}{0} \\
\tilde{0} \\
0 \\
0\end{array}$ & 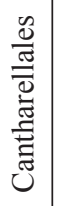 & 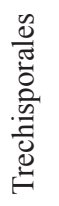 & $\begin{array}{l}\frac{0}{0} \\
\frac{0}{0} \\
\frac{0}{0} \\
\frac{0}{0} \\
\frac{0}{0} \\
\text { की }\end{array}$ & $\begin{array}{l}\frac{v}{0} \\
\frac{0}{0} \\
\frac{0}{0} \\
: \frac{0}{0} \\
\frac{0}{0} \\
\text { की }\end{array}$ & $\begin{array}{l}\frac{0}{0} \\
\frac{\pi}{0} \\
\frac{2}{2} \\
0\end{array}$ & 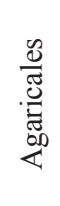 & 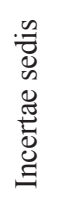 & 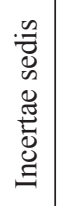 & $\begin{array}{l}\frac{n}{0} \\
\frac{0}{0} \\
0 \\
\frac{2}{0} \\
0 \\
0\end{array}$ & 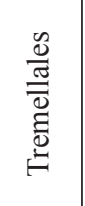 & 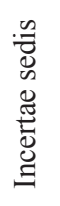 & 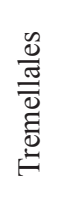 & 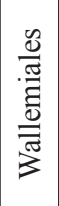 \\
\hline
\end{tabular}

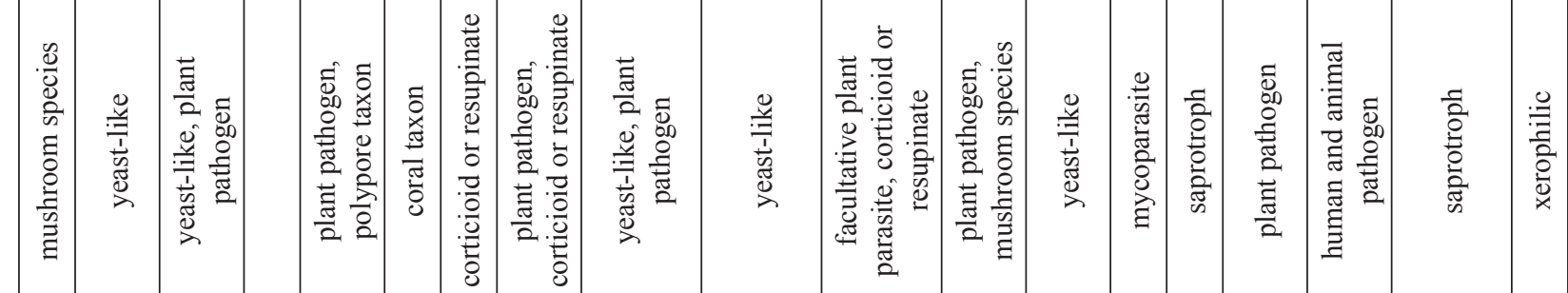

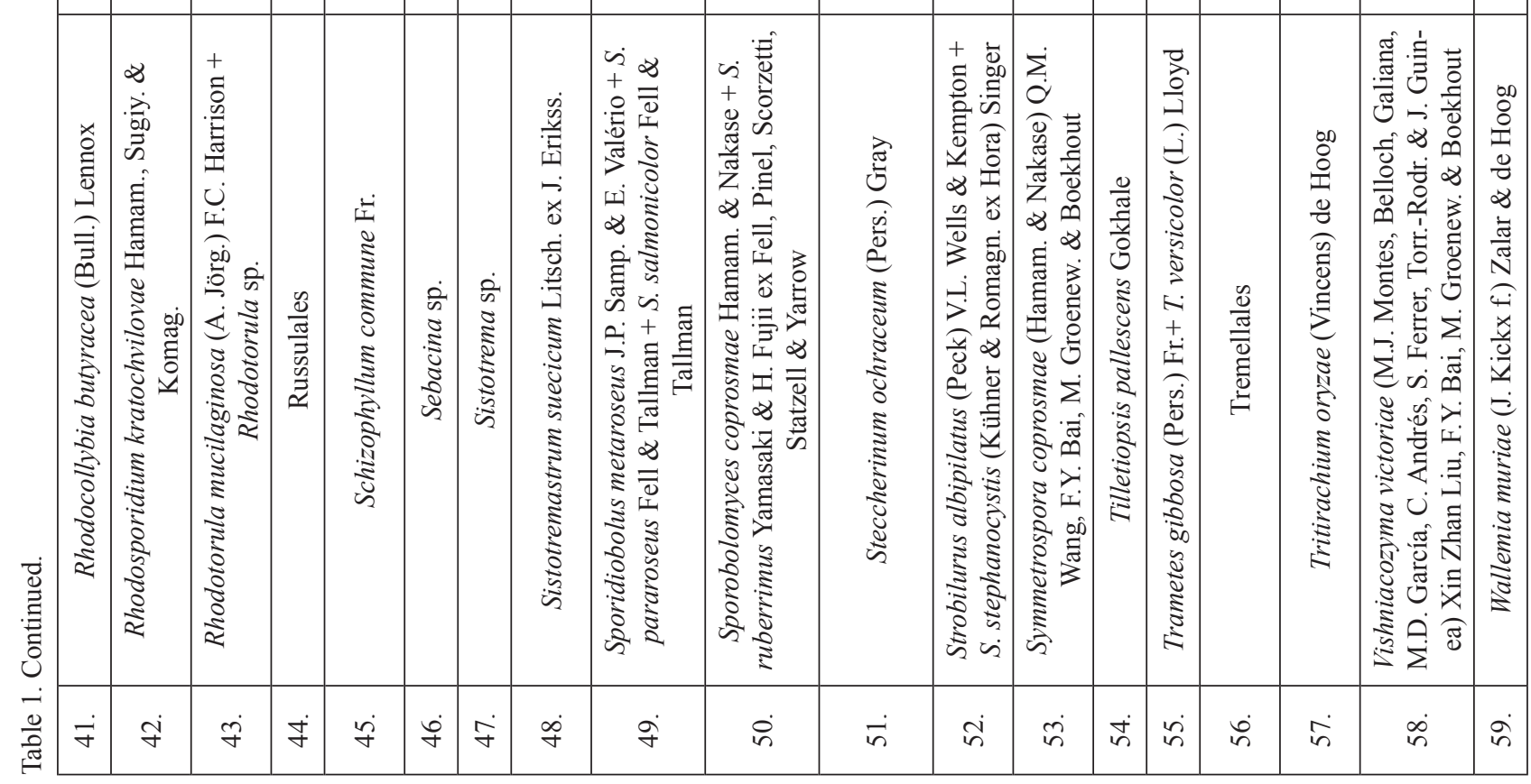




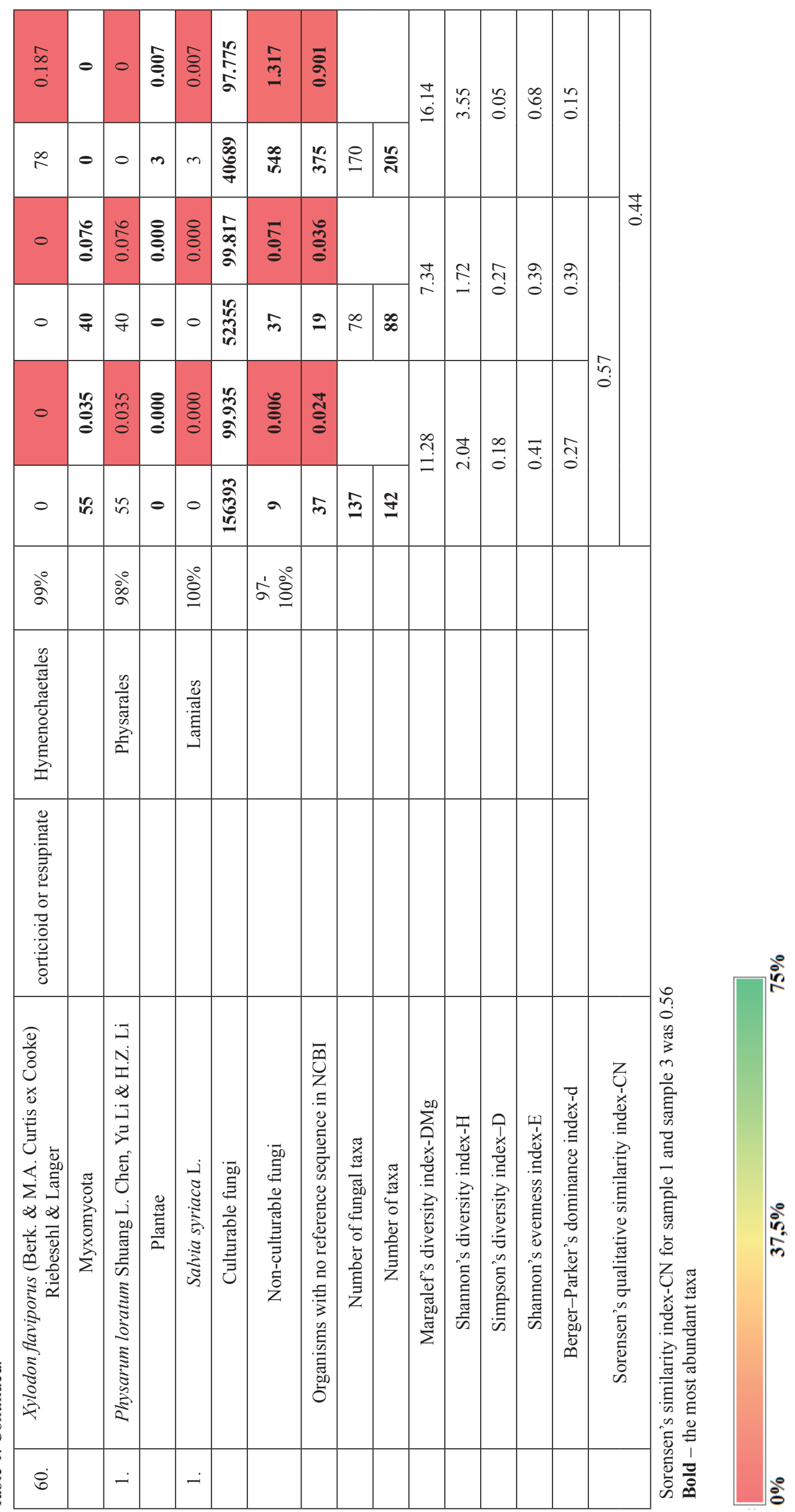


by $1,8,131$ and 83 taxa, respectively. Non-culturable organisms were represented by 70 taxa. More than $90 \%$ of taxa were identified to genus or species. There were 142 and 205 taxa in parchment (samples 1 and 3) and 88 taxa in paper (sample 2). The number of species shared by parchment and paper from a single archive in Torun was 9, and by the two parchments from two different archives (Toruń and Warsaw) was 33 (Fig. 1). The number of species shared by all three samples was 61 , and the numbers of taxa separate for samples 1,2 and 3 were respectively 36, 1 and 85 .

Fungal communities consisted of the few abundant taxa and many rare taxa. The most frequent were Ascomycota from the genera Acremonium, Alternaria, Aspergillus, Beauveria, Bionectria, Botrytis, Chaetomium, Penicillium and Trichoderma. The most common were Chaetomium murorum, Chaetomium globosum and Chaetomium iranianum.

Communities included plant pathogens, epiphytes or endophytes of a wide range of plants, and possibly facultative plant pathogens, saprotrophs known from soil, decaying leaves, needles, wood (soft, white and brown rot fungi) and other plant material, human, animal and nematode pathogens, entomopathogenic taxa with worldwide distribution and a relatively wide host range, mycoparasites, white and black yeast-like taxa, taxa with medicinal properties, lichenized fungi, food contaminants, indoor contaminants, taxa known from herbivore dung, keratin-degrading taxa, mushroomproducing taxa, corticioid, resupinate, polypore, coral annual or perennial taxa, taxa with antagonistic properties, xerophilic taxa and endangered fungi included in the Red List of Threatened Species. The mycobiota of parchment, compared with that of paper, had: (i) more diversity, indicated by $\mathrm{DMg}$ (16.14 and 11.28 versus 7.34$), \mathrm{H}$ (3.55 and 2.04 versus 1.72$)$ and D (0.05 and 0.18 versus 0.27 ); (ii) more even distribution of species, indicated by $\mathrm{E}$ ( 0.68 and 0.41 versus 0.39 ); and (iii) less dominance of single species, indicated by $\mathrm{d}(0.15$ and 0.27 versus 0.39$)$. There was more similarity in communities on paper and parchment stored in one place (Toruń) $(\mathrm{CN}=0.57)$ and on parchment stored in different places $(\mathrm{CN}=0.56)$ than on paper and parchment stored in two different places $(\mathrm{CN}=0.44)$ (Table 1).

\section{Discussion}

Historical paper or parchment documents should be stored in a cool, stable environment - ideally at $20^{\circ} \mathrm{C}$ and $50 \%$ relative humidity in a well-insulated and ventilated room, with no direct daylight or artificial light, in an acid- and lignin-free storage box. Such conditions had not always been provided for the documents studied. The torah, in particular, had been moved continually, used during religious ceremonies, and stored in the past in accidental places in non-controlled conditions.
This may explain its advanced deterioration and high microbial contamination.

A non-destructive and non-invasive method for quantitative characterization of deterioration, based on spectral measurements, was applied to evaluate the scale of damage of the studied documents. The collagen-to-gelatin ratio, which can be estimated from the synchronous fluorescence spectra of modern and historical samples and of pure collagen and gelatin data, was applied for characterization of parchment condition. Analysis of fluorescence peaks and their comparison with peaks of other studies indicated the moderate stage of chemical deterioration of the studied documents. If there was at least 225 fungal taxa detected on both documents, their state seemed to result partly from fungal colonization.

The mycobiota of historical parchment documents has not received as much attention as the mycobiota of paper documents. Studies of parchment and leather have concentrated mostly on their physical damage and chemical changes and only rarely on their microbial colonization $[1,5,7,15,16]$. The greater diversity of mycobiota on parchment compared with paper (170 and 137 versus 87 taxa) supports earlier findings [6]. Studies emphasize a parchment's vulnerability to fungal infestation and colonization, resulting from its chemical properties. Parchment contains proteins ( $\sim 95 \%$ collagen) and some lipids (glycerides). With their low $\mathrm{pH}$, both are ideal sources of nutrients for fungi. The preparation of parchment includes treatment with lime, which neutralizes the excessive acidity. Additional compounds (e.g., gum Arabic used for repair of document 1) might be additional sources of specific nutrients, increasing the spectrum of microorganisms.

The vulnerability of parchment to fungal infestation can also result from its extremely strong response to environmental variation. It deforms and deteriorates with the slightest change in temperature and humidity. Physical changes may be followed by chemical degradation; in high humidity the collagen fibres lose their initial high thermal and mechanical stability and convert to gelatin, which can easily be degraded by many fungal taxa, including Alternaria alternata, Aspergillus spp., Mucor plumbeus, Penicillium chrysogenum, Phoma herbarum and Trichoderma longibrachiatum detected by us and others [29, 30]. Aspergillus versicolor and Penicillium chrysogenum are among the most efficient, degrading $25-30 \%$ of gelatin in 2-3 weeks after colonization [16, 30].

The number of taxa presented here, detected with the Illumina technique applied, was much higher than in other studies, when only nine species from Aspergillus, Mucor, Phoma and Penicillium genera in nine historical parchment documents were detected with multiphasic approach applied, various sampling procedures and different microbiological methods [5], and 42 species (mostly from Alternaria, Cladosporium, Epicoccum and Penicillium genera) were detected with classical and molecular methods [15]. 
The most frequent taxa detected belonged to the Ascomycota. Often they are ubiquitous fungi present in human habitations due to intensive reproduction, easy dispersal, strong degrading properties and wide habitat range $[5,7,10,15,16,31,32]$. They usually have cellulolytic and proteolytic properties [5, 33-37]. Basidiomycota occurred more rarely. The proteolytic activity of Basidiomycota has been confirmed in the genera of Armillaria, Cantharelus, Russula and Schizophyllum [38]. The number and diversity of proteases produced by these taxa seem to be remarkable. Proteolysis by other taxa among those detected can also be expected, even though it has not yet been studied or proven. Production of proteases in Basidiomycota, however, seems to be regulated by the carbon and nitrogen sources and $\mathrm{C}: \mathrm{N}$ ratio and activated at low levels of compounds containing accessible nitrogen [39, 40]. Parchment rich in nitrogen therefore may not be the best substrate for Basidiomycota.

Some taxa detected were more or less specialized. One of those is Chaetomium, which occurred in Document 1 with $53.12 \%$ and $73.07 \%$ frequency. The fungus is a specialist colonizer of leather and skin due to its utilization of collagen and keratin. Its dominance can also result from: (i) successful survival during longdistance travels, (ii) coping with extreme conditions at high elevations, (iii) resistance to antifungal substances produced by neighbours, and (iv) the production of antimicrobial compunds [41-43].

We recorded a few species of Cladosporium, but they were less frequent than in other studies. The most common Penicillium was Penicillium chrysogenum, which was also the predominant species on other parchment documents $[5,16]$. Another Penicillium, i.e., $P$. decumbens, was common in one study [16] but rare in ours.

Acremonium charticola, Beauveria bassiana, Bionectria rossmaniae, Botrytis cinerea, Chaetomium murorum, Fusarium armeniacum, Fusarium avenaceum, Fusarium sporotrichioides, Malassezia globosa, Mortierella spp., Sarocladium strictum, Serpula himantioides, Sordaria fimicola, Strasseria geniculata, and most species of Aspergillus and Penicillium recorded had never been isolated previously from parchment documents. Bjerkandera adusta and Phoma herbarum were detected only recently [5].

Many of the species detected occur naturally as epiphytes on a wide range of plants. Some, however, are known from their extreme habitats. Candida sake, Cystofilobasidium macerans, Leucosporidiella creatinivora, Mortierella spp., Naganishia adeliensis, Tetracladium, Thelebolus ellipsoideus and Volucrispora graminea prefer moist and cold habitats [44]. Exophiala xenobiotica is known for habitats rich in monoaromatic hydrocarbons and alkanes. Naganishia spp. and Wallemia spp. are flexible 'opportunitrophs' known from the most extreme terrestrial habitats on Earth [45].

The group of the most unexpected and surprising taxa included: (i) Arachniotus aurantiacus, Candida boleticola, and Debaryomyces prosopidis, known from dung, mushrooms and exudates of mesquite trees, respectively; (ii) Candida sake, Naganishia adeliensis, and Vishniacozyma victoriae, known from seawater, decaying algae, mosses, lichens and soil in extremely cold, Arctic and Antarctic habitats [44, 46]; (iii) Leptospora rubella and Phaeococcomyces eucalypti, known from living leaves and leaf litter of Eucalyptus, and (iv) Pleurophoma ossicola and Rhodotorula mucilaginosa, known from bone and human beings [47]. The presence of taxa known from remote locations or non-native plants can be explained by fungal mobility. Microorganisms can be transported over long distances. Biogeographic patterns of microbial community structure show widespread long-distance dispersal on a global scale [48-50].

Some of the fungi detected may be potential opportunistic pathogens in immunocompromised patients. They may cause allergies, asthma, infections of the eye, ear, skin, nails, sinus, lungs, joints, bones, brain or the central nervous system [51-53]. Extracellular proteolytic enzymes may contribute to their pathogenicity on humans [33].

There is, however, an example of a beneficial, commensal human-fungus relationship involving Malassezia globose, which has been detected in both documents. Though the fungus seems to play a pathogenic role in several dermatologic conditions, its proteases may hydrolyse Staphylococcus aureus protein A - an important virulence factor involved in immunity evasion and infection on human skin [37].

Mycological analyses of archived documents often give different results depending on the character and age of the substrate, conditions of storage, inter-species relationships and interactions, and techniques used $[2,4,7]$. Older collections are generally colonized by higher numbers of fungi [15]. Exposure to light, suitable $\mathrm{pH}$, emissions, pollutants including ozone, and volatile organic and inorganic compounds are vital [54]. Each document has its own history and its own specific contaminants. Each item should therefore be considered as a unique example with its own specific characteristics.

The culture-independent method used in this study contributes to a more complete survey of the mycobiota. This approach does not, however, give any information on the functional role of a particular taxon. The Illumina technique used based on rDNA gene detection is unable to give any information on the biodegradative potential of fungi detected and hazard connected with their presence in an archive. The molecular analysis used might have referred to DNA fragments that do not belong to microorganisms currently present. The method does not explain whether particular taxa can grow in parchment or occur only in the form of dormant propagules that fall onto the document from the air. Therefore, while the analysis has identified significant and potentially important diversity of fungi, it remains incomplete without information on their activity. 
Analysis of both diversity and activity of fungi would be possible using transcriptomic technologies that would provide a broad account of active and dormant cellular processes.

\section{Conclusions}

Old written documents or other items can have priceless historical value to our cultural heritage, whether used for communication or religious purposes. Studies of biodeterioration-related fungi in documents and religious objects are important for understanding the scale of the biodeterioration process, and the necessity for and methods of conservation.

\section{Acknowledgements}

The authors are grateful to Prof. Elżbieta Jabłońska from the Department of Paper and Leather Conservation, Nicolaus Copernicus University in Torun, Poland, for her kind collaboration.

\section{Conflict of Interest}

The authors declare no conflict of interest.

\section{References}

1. KARBOWSKA-BERENT J., STRZELCZYK A.B. The role of Streptomycetes in the biodeterioration of historic parchment. Copernicus University Press, 2000.

2. MICHAELSEN A., PIÑAR G., PINZARI F. Molecular and microscopic investigation of the microflora inhabiting a deteriorated Italian manuscript dated from the $13^{\text {th }}$ century. Microb. Ecol. 60, 69, 2010.

3. PIÑAR G., PINZARI F., STERFLINGER K. Modern technologies as basis for the preservation of parchment. In: López Montes A.M., Collado Montero F., Medina Flórez V., Espejo Arias T., García Bueno A. (Eds) $18^{\text {th }}$ International meeting on heritage conservation. 250, 2011.

4. PRINCIPI P., VILLA F., SORLINI C., CAPPITELLI F. Molecular studies of microbial community structure on stained pages of Leonardo da Vinci's Atlantic Codex. Microb. Ecol. 61, 214, 2011.

5. KRAKOVA L., CHOVANOVÁ K., SELIM S.A., ŠIMONOVIČOVÁ A., PUŠKAROVÁ A., MAKOVÁ A., PANGALLO D. A multiphasic approach for investigation of the microbial diversity and its biodegradative abilities in historical paper and parchment documents. Int. Biodeter. Biodegr. 70, 117, 2012.

6. PINZARI F.,CIALEI V.,PIÑAR G. A case study of ancient parchment biodeterioration using variable pressure and high vacuum scanning electron microscopy. Historical technology, Materials and conservation: SEM and microanalysis. In: Meeks N., Cartwright C., Meek A., Mongiatti A. (Eds) Archetype Publications - International Academic Projects,1 Birdcage Walk, London. 2012.
7. MESQUITA N., PORTUGAL A., VIDEIRA S., RODRÍGUEZ-ECHEVERRÍA S., BANDEIRA A.M.L, SANTOS M.J.A., FREITAS H. Fungal diversity in ancient documents: a case study on the archive of the University of Coimbra. Int. Biodeter. Biodegr. 63, 626, 2009.

8. PINZARI F., MONTANARI M. Mould growth on library materials stored in compactus-type shelving units (Chapter 11) In: Abdul-Wahab A., 1-Sulaiman S.A. (Eds) Sick building syndrome in public buildings and workplaces. Burlington. Elsevier. 2011.

9. PINZARI F., COLAIZZI P., MAGgI O., PERSIANI A.M., SCHÜTZ R., RABIN I. Fungal bioleaching of mineral components in a twentieth-century illuminated parchment. Anal. Bioanal. Chem. 402, 1541, 2012.

10. STERFLINGER K., PINZARI F. The revenge of time: fungal deterioration of cultural heritage with particular reference to books, paper and parchment. Environ. Microb. 14 (3), 559, 2012.

11. NUNES I., MESQUITA N., CABOVERDEA S., LEITÃOBANDEIRAC A.M., CAROLINOD M.M., PORTUGAL A., BOTELHO M.L. Characterization of an airborne microbial community: a case study in the archive of the University of Coimbra, Portugal. Int. Biodeter. Biodeg. 79, 36, 2013.

12. TROIANO F., POLO A., VIlla F., CAPPITELli F. Assessing the microbiological risk to stored sixteenth century parchment manuscripts: a holistic approach based on molecular and environmental studies. Biofouling 30 (3), 299, 2014.

13. PIÑAR G., STERFLINGER K., ETTENAUER J., QUANDT A., PINZARI F. A combined approach to assess the microbial contamination of the Archimedes palimpsest. Microb. Ecol. 69, 118, 2015.

14. PIÑAR G., STERFLINGER K., PINZARI F. Unmasking the measles-like parchment discoloration: molecular and microanalytical approach. Environ. Microb. 17, 427, 2015.

15. DE CARVALHO H.P, MESQUITA N., TROVÃO J., DA SILVA J.P, ROSA B., MARTINS R., BANDEIRA A.M.L., PORTUGAL A. Diversity of fungal species in ancient parchments collections of the Archive of the University of Coimbra. Int. Biodeter. Biodegr. 108, 57, 2016.

16. LECH T. Evaluation of a parchment document, the $13^{\text {th }}$ century incorporation charter for the City of Krakow, Poland, for microbial hazards. Appl. Environ. Microb. 82 (9), 2620, 2016.

17. ARAI H. Foxing caused by fungi: twenty-five years of study. Int. Biodeter. Biodegr. 46, 181, 2000.

18. MONTEMARINI-CORTE A., FERRONI A., SALVO V.S. Isolation of fungal species from test samples and maps damaged by foxing, and correlation between these species and the environment. Int. Biodeter. Biodegr. 51, 167, 2003.

19. STERFLINGER K., PINZARI F. Microbial deterioration of cultural heritage and works of art - tilting at windmills? Appl. Microbiol. Biot. 97, 9637, 2013.

20. KARBOWSKA-BERENT J. disinfection of paper-based cultural property. Wydawnictwo Naukowe UMK, Toruń. 2014 [In Polish].

21. MICHAELSEN A., PINZARI F., RIPKA K., LUBITZ W., PIÑAR G. Application of molecular techniques for identification of fungal communities colonizing paper material. Int. Biodeter. Biodegr. 58, 133, 2006.

22. DOLGIN B., BULATOV V., SCHECHTE I. Nondestructive assessment of parchment deterioration by optical methods. Anal. Bioanal. Chem. DOI 10.1007/ s00216-007-1410-0. 2007. 
23. IHRMARK K., BÖDEKER I.T., CRUZ-MARTINEZ K., FRIBERG H., KUBARTOVA A., SCHENCK J., STRID Y., STENLID J., BRANDSTRÖM-DURLING M., CLEMMENSEN K.E., LINDAHL B.D. New primers to amplify the fungal ITS2 region - evaluation by 454 -sequencing of artificial and natural communities. FEMS Microbiol. Ecol. 82 (3), 666, 2012.

24. GWEON H.S., OLIVER A., TAYLOR J., BOOTH T., GIBBS M., READ D.S., GRIFFITHS R.I., SCHONROGGE K. PIPITS: An automated pipeline for analyses of fungal internal transcribed spacer sequences from the Illumina sequencing platform. Methods Ecol. Evol. 6 (8), 973, 2015.

25. ZHANG J., KOBERT K., FLOURI T., STAMATAKIS A. PEAR: A fast and accurate Illumina Paired-End reAd mergeR. Bioinformatics 30 (5), 614, 2014.

26. BENGTSSON-PALME J., VELDRE V., RYBERG M., HARTMANN M., BRANCO S., WANG Z., GODHE A., BERTRAND Y., DE WIT P., SANCHEZ M., EBERSBERGER I., SANLI K., DE SOUZA F., KRISTIANSSON E., ABARENKOV K., ERIKSSON K.M., NILSSON R.H. Improved software detection and extraction of ITS1 and ITS2 from ribosomal ITS sequences of fungi and other eukaryotes for analysis of environmental sequencing data. Methods Ecol. Evol. 4, 914, 2013.

27. COLE J.R., WANG Q., FISH J.A., CHAI B., MCGARRELL D.M., SUN Y., BROWN C.T., PORRASALFARO A., KUSKE C.R., TIEDJE J.M. Ribosomal Database Project: data and tools for high throughput rRNA analysis. Nucleic Acids Res. 42 (Database issue). D633-D642, 2014.

28. MAGURRAN A.E. Ecological diversity and its measurement. Princeton, NJ. Princeton University Press. 1988.

29. LEONHARTSBERGER S., LAFFERTY R.M., KORNETI L. Use of collagen hydrolysate as a complex nitrogen source for the synthesis of penicillin by Penicillium chrysogenum. J. Biotechnol. 30, 299, 1993.

30. ABRUSCI C., MARQUINA D., DEL AMO A., CATALINA F. Biodegradation of cinematographic gelatin emulsion by bacteria and filamentous fungi using indirect impedance technique. Int. Biodeter. Biodegr. 60, 137, 2007.

31. KARBOWSKA-BERENT J., GÓRNY R.L., STRZELCZYK A.B., WLAZŁO A. Airborne and dust borne microorganisms in selected Polish libraries and archives. Build. Environ. 46, 1872, 2011.

32. SKÓRA J., GUTAROWSKA B., PIELECHPRZYBYLSKA K., STĘPIEŃ Ł., PIETRZAK K., PIOTROWSKA M., PIETROWSKI P. Assessment of microbiological contamination in the work environments of museums, archives and libraries. Aerobiologia (Bologna) 31 (3), 389, 2015.

33. KREDICS L., ANTAL Z., SZEKERES A., MANCZINGER L., DÓCZI I., KEVEI F., NAGY E. Production of extracellular proteases by human pathogenic Trichoderma longibrachiatum strains. Acta Microbiol. Imm. Hung. 51 (3), 283, 2004.

34. PANGALLO D., KRAKOVÁ L., CHOVANOVÁ K., BUČKOVA M., PUŠKAROVÁ A., ŠIMONOVIČOVÁ A. Disclosing a crypt: microbial diversity and degradation activity of the microflora isolated from funeral clothes of Cardinal Peter Pazmany. Microbiol. Res. 168, 289, 2013.

35. ZAFERANLOO B., QUANG T.D., DAUMOO S., GHORBANI M.M., MAHON P.J.,PALOMBO E.A. Optimization of protease production by endophytic fungus, Alternaria alternata, isolated from an Australian native plant. World J. Microb. Biot. 30 (6), 1755, 2014.

36. BEHERA B.C., SETHI B.K., MISHRA R.R., DUTTA S.K.,THATOI H.N. Microbial cellulases - Diversity \& biotechnology with reference to mangrove environment: A review. J. Gen. Engin. Biotechn. 15, 197, 2017.

37. LI H., GOH B.N., TEH W.K., JIANG Z., GOH J.P.Z., GOH A., WU G., HOON S.S., RAIDA M., CAMATTARI A., YANG L., O'DONOGHUE A.J., DAWSON T.L. JR. Skin commensal Malassezia globosa decreted protease attenuates Staphylococcus aureus biofilm formation. J. Invest. Dermatol. 138 (5), 1137, 2018.

38. JOHNSTON J.M., RAMOS E.R., BILBREY R.E., GATHMAN A.C., LILLY W.W. Characterization of ScPrI, a small serine protease, from mycelia of Schizophyllum commune. Mycol. Res. 104, 726, 2000.

39. SATO S., LIU F., KOC H., TIEN M. Expression analysis of extracellular proteins from Phanerochaete chrysosporium grown on different liquid and solid substrates. Microbiology 153, 3023, 2007.

40. VASINA D.V., PAVLOV A.R.,KOROLEVA O.V Extracellular proteins of Trametes hirsuta st. 072 induced by copper ions and a lignocellulose substrate. BMC Microbiology 16 (1), 106, 2016.

41. SERENA C., ORTONEDA M., CAPILLA J., PASTOR F.J., SUTTON D.A., RINALDI M.G., GUARRO J. In vitro activities of new antifungal agents against Chaetomium spp. and inoculum standardization. Antimicrob. Agents Chemother. 47, 3161, 2003.

42. MCMULLIN D.R., SUMARAH M.W., MILLER J.D. Chaetoglobosins and azaphilones produced by Canadian strains of Chaetomium globosum isolated from the indoor environment. Mycotoxin Res. 29, 47, 2013.

43. MCMULLIN D.R., SUMARAH M.W., BLACKWELL B.A., MILLER J.D. New azaphilones from Chaetomium globosum isolated from the built environment. Tetrahedron Lett. 54 (6), 568, 2013.

44. BALLESTER-TOMÁS L, PRIETO JA, GIL J.F., BAEZA M., RANDEZ-GIL F. The Antarctic yeast Candida sake: Understanding cold metabolism impact on wine. Int. J. Food Microbiol. 245, 59, 2017.

45. CHMIDT S.K., VIMERCATI L., DARCY J.L., ARÁN P., GENDRON E.M.S., SOLON A.J., PORAZINSKA D., DORADOR C. A Naganishia in high places: functioning populations or dormant cells from the atmosphere? Mycology 8, 153, 2017.

46. LIU X.Z., WANG, Q.M., GÖKER M., GROENEWALD M., KACHALKIN A.V., LUMBSCH H.T., MILLANES A.M., WEDIN M., YURKOV A.M., BOEKHOUT T., BAI F.Y. Towards an integrated phylogenetic classification of the Tremellomycetes. Stud. Mycol. 81, 85, 2015.

47. CROUS P.C., SHIVAS R.G., WINGFIELD M.J., SUMMERELL B.A., ROSSMAN A.Y., ALVES J.L., ADAMS G.C., BARRETO R.W., BELL A., COUTINHO M.L., FLORY S.L., GATES G., GRICE K.R., HARDY G.E.ST.J, KLECZEWSKI N.M., LOMBARD L., LONGA C.M.O., LOUIS-SEIZE G., MACEDO F., MAHONEY D..P, MARESI G., MARTIN-SANCHEZ P.M., MARVANOVÁ L., MINNIS A.M., MORGADO L.N., NOORDELOOS M.E., PHILLIPS A.J.L., QUAEDVLIEG W., RYAN P.G., SAIZ-JIMENEZ C., SEIFERT K.A., SWART W.J.,TAN Y.P., TANNEY J., THU P.Q., VIDEIRA S.I.R., WALKER D.M., GROENEWALD J.Z. Fungal Planet description sheets: 128-153. Persoonia 29, 146, 2012. 
48. BARBERÁN A., HENLEY J., FIERER N.,CASAMAYOR E.O. Structure, inter-annual recurrence, and global-scale connectivity of airborne microbial communities. Sci. Total Environ. 487, 187, 2007.

49. DARCY J.L., LYNCH R., KING A.J., ROBESON M.S., SCHMIDT S.K. Global distribution of Polaromonas phylotypes - evidence for a highly successful dispersal capacity. PLoS One.6, 23742, 2011.

50. ITANI G.N., SMITH C.A. Dust rains deliver diverse assemblages of microorganisms to the Eastern Mediterranean. Sci. Rep. 6, 22657, 2016.

51. DE HOOG G.S., GUARRO J., GENE J., FIGUERAS M.J. Atlas of Clinical Fungi. Centraalbureau voor Schimmelcultures, Utrecht/Universitat Rovira i Virgili, Reus, Spain. 2001.
52. TSIODRAS S., PAPAGEORGIOU S., MELETIADIS J., TOFAS P., PAPPA V., PANAYIOTIDES J., KARAKITSOS P., ARMAGANIDIS A., PETRIKKOS G. Rhodotorula mucilaginosa associated meningitis: A subacute entity with high mortality. Med. Mycol. Case Rep. 6, 46, 2014.

53. SCHEUFEN S., STROMMER D.S., WEISENBORN D.J., PRENGER-BERNINGHOFF E., THOM N., BAUER N., KÖHLER K., EWERS C. Clinical manifestation of an amelanotic Sporothrix schenckii complex isolate in a cat in Germany. JMM Case Reports. 2015.

54. PINHEIRO A.C., VIEGAS C., VIEGAS S., VERÍSSIMO C., BRANDÃO J., MACEDO M.F. Indoor air quality in Portuguese archives: a snapshot on exposure levels. J. Toxicol. Env. Heal. A 75, 1359, 2012. 
